\title{
An all-Mach method for the simulation of bubble dynamics problems in the presence of surface tension
}

\author{
Daniel Fuster, Stéphane Popinet \\ Sorbonne Université, Centre National de la Recherche Scientifique, UMR 7190, Institut \\ Jean Le Rond D'Alembert, F-75005 Paris, France
}

\begin{abstract}
This paper presents a generalization of an all-Mach formulation for multiphase flows accounting for surface tension and viscous forces. The proposed numerical method is based on the consistent advection of conservative quantities and the advection of the color function used in the Volume of Fluid method avoiding any numerical diffusion of mass, momentum and energy across the interface during the advection step. The influence of surface tension and liquid compressibility on the dynamic response of the bubbles is discussed by comparing the full 3D solution with the predictions provided by the Rayleigh-Plesset equation for two relevant problems related to the dynamic response of bubbles to pressure disturbances: The linear oscillation of a single bubble in an acoustic field and the Rayleigh collapse problem. Finally, the problem of the collapse of a bubble close to a wall is compared with experimental results showing the robustness of the method to simulate the collapse of air bubbles in liquids in problems where bubbles generate a high velocity liquid jet.
\end{abstract}

Keywords: All-Mach formulation, bubble dynamics, multiphase flows, compressible flows, volume-of-fluid method.

\section{Introduction}

The simulation of bubbles accounting for compressible effects is a topic of interest in various applications related to underwater explosions [1,2], engineering applications $[3,4]$ or medical treatment such as HIFU or lithotripsy $[5,6]$. In all these processes it is crucial to understand the dynamic response 
of bubbles to pressure waves motivating the development of numerical methods for the Direct Numerical Simulation of bubbles in slightly compressible liquids [7].

Despite the development of numerical techniques to solve the NavierStokes equations in the presence of multiple phases $[8,5]$ there are still numerical difficulties when coupling compressibility, viscous and surface tension effects [9]. For instance classical solvers for compressible flows based on Riemann solvers rarely take into account surface tension [10]. This is due to the intrinsic difficulties related to the treatment of sharp interfaces and also how to deal with the jump of properties across the interface ensuring that surface tension terms satisfy basic relations such as the balance between pressure and capillary forces in an equilibrium state [11].

One family of numerical methods capturing compressibility effects resort to the generalization of numerical schemes developed for incompressible fluids $[12,13,14,15,16,17]$. Among these methods, all-Mach semi-implicit formulations are especially appealing as they have shown to provide accurate results in different situations ranging from pure incompressible flows to compressible problems involving the presence of shock waves. One of the advantage of these methods is that they allow for a natural extension of the numerical strategies developed for incompressible multiphase flows, which have been extensively developed and tested in problems where surface tension forces are relevant. In addition, these methodologies avoid the classical time step restriction defined by the speed of sound of the least-compressible fluid, which is convenient for the simulation of subsonic flows that combine substances with very different compressibility. This is typically the case in bubbly flows where the gas compressibility is much larger than that of the liquid, sometimes considered as an incompressible substance.

In this work we present an extension of the all-Mach semi-implicit solver using the Volume-Of-Fluid (VOF) method including surface tension and viscous forces. The main advantages of VOF methods is that they keep the representation of the interface sharp, they enforce volume conservation in the incompressible limit and handle naturally topological changes of the interface. The method takes into account viscous and surface tension effects by generalizing the classical formulation used in previous incompressible solvers 
[18]. The extended formulation accounts for fluids with arbitrary values of the thermal dilatation coefficient, which is typically not the case in previous works. We chose the Basilisk solver [19] as a platform to implement the new numerical scheme. The methods implemented in the code have been well validated for the simulation of multiphase flows in various problems involving interfaces [20]. Moreover, the method has been designed to reduce the appearance of parasitic currents induced by surface tension forces [18], which is an appealing characteristic of the numerical scheme if one wants to reduce any artificial energy dissipation/generation in the system.

The manuscript is structured as follows: The basic equations are presented first. Then we describe the numerical methods used to solve the

equations. Two numerical tests for single phase flows are introduced to discuss the properties of the method in two limiting situations: the linear propagation of pressure waves and the propagation of shock waves. Then, we quantify the numerical oscillations induced by surface tension forces in an equilibrium state and the velocity and pressure oscillations generated by the advection of an interface before discussing various tests proposed to assess the capability of the method to capture the dynamic response of bubbles to pressure changes. Finally the problem of the collapse near a wall is used to show the robustness of the solver before presenting the conclusions.

\section{Basic equations}

In this work we solve for the Navier-Stokes equations for a mixture of two immiscible substances where the position is defined by a Heaviside function $\mathcal{H}$ that takes the value of 1 in the reference phase [21]. The interface position is then given by the solution of an advection equation for $\mathcal{H}$

$$
\frac{\partial \mathcal{H}}{\partial t}+\boldsymbol{u} \cdot \nabla \mathcal{H}=0 .
$$

The continuity and momentum equation for the $i$ th phase are

$$
\begin{array}{r}
\frac{\partial \rho_{i}}{\partial t}+\nabla \cdot\left(\rho_{i} \boldsymbol{u}_{i}\right)=0, \\
\frac{\partial \rho_{i} \boldsymbol{u}_{i}}{\partial t}+\nabla \cdot\left(\rho_{i} \boldsymbol{u}_{i} \boldsymbol{u}_{i}\right)=-\nabla p_{i}+\nabla \cdot \tau_{i}^{\prime},
\end{array}
$$


where $\rho$ is the density, $\boldsymbol{u}$ is the fluid velocity vector, $p$ is the fluid pressure and $\tau^{\prime}=\mu\left(\nabla \boldsymbol{u}+(\nabla \boldsymbol{u})^{T}\right)-\frac{2}{3} \mu \nabla \cdot \boldsymbol{u} \delta$ is the viscous stress tensor depending on the dynamic viscosity $\mu$.

The equations between the two phases can be weighted by the respective volume fraction and then added to obtain evolution equations for the various averaged quantities. To do this it is important to account for the relation between the velocity and pressure established by mass and momentum balances across a differentially thin interface located at $\boldsymbol{x}_{I}$. In the absence of mass transfer the velocity is continuous $\boldsymbol{u}_{1}\left(\boldsymbol{x}_{I}\right)=\boldsymbol{u}_{2}\left(\boldsymbol{x}_{I}\right)$ and therefore it is natural to work with a single continuous averaged velocity field $\boldsymbol{u}$. The Laplace equation gives the pressure jump at the interface as

$$
p_{1}-p_{2}=\sigma \kappa
$$

where $\sigma$ is the surface tension coefficient and $\kappa$ is the curvature of the interface. Note that following classical formulations for incompressible multiphase flows we have neglected the viscous stress jump across the interface. Applying these conditions for the cells containing an interface we can write the momentum equation for the averaged quantities as [22]

$$
\frac{\partial \rho \boldsymbol{u}}{\partial t}+\nabla \cdot(\rho \boldsymbol{u u})=-\nabla p+\nabla \cdot \tau_{i}^{\prime}+\sigma \kappa \nabla c .
$$

When accounting for compressibility we aditionally solve an equation for the energy. In this paper we restrict ourselves to situations where thermal diffusion and mass transfer effects are not relevant (e.g. high frequency oscillations [23]), so that the total energy equation is

$$
\frac{\partial \rho_{i} e_{i}+1 / 2 \rho_{i} \boldsymbol{u}_{i}^{2}}{\partial t}+\nabla \cdot\left(\rho_{i} e_{i} \boldsymbol{u}_{i}+1 / 2 \rho_{i} \boldsymbol{u}_{i}^{2}\right)=-\nabla \cdot\left(\boldsymbol{u}_{i} p_{i}\right)+\nabla \cdot\left(\tau_{i}^{\prime} \boldsymbol{u}_{i}\right),
$$

where $e$ denotes the internal energy. Alternative forms of the energy equation include the internal energy equation, which in the absence of thermal diffusion takes the form

$$
\rho_{i} c_{p, i} \frac{D T_{i}}{D t}=\beta_{T, i} T_{i} \frac{D p_{i}}{D t}+\Phi_{v, i}
$$

where $T$ is temperature, $c_{p, i}$ is the specific heat measured at constant pressure, $\beta_{T, i}$ is the thermal dilatation coefficient and $\Phi_{v, i}=\nabla \boldsymbol{u}: \tau^{\prime}$ is the 
viscous dissipation function. To obtain an evolution equation for pressure we follow the procedure proposed in [24], where the internal energy (Eq. 7) and the continuity equation (Eq. 2) are combined to express the divergence of the velocity as a function of pressure. To do that we obtain the differences between two thermodynamic states as a sum of the changes of density in an isothermal process plus the density change in an isobaric process

$$
d \rho=\left(\frac{\partial \rho}{\partial p}\right)_{T} d p+\left(\frac{\partial \rho}{\partial T}\right)_{p} d T,
$$

which can be written using the definitions of the speed of sound, polytropic coefficient and thermal dilatation coefficient as

$$
d \rho=\frac{\gamma}{c^{2}} d p-\rho \beta d T
$$

Using Eqs. 2, 7, 9 we obtain

$$
\frac{1}{\rho c_{\mathrm{eff}}^{2}} \frac{D p}{D t}-\frac{\beta_{T} \Phi_{v}}{\rho c_{p}}=-\nabla \cdot \boldsymbol{u},
$$

where

$$
\frac{1}{\rho c_{\mathrm{eff}}^{2}}=\left[\frac{\gamma}{\rho c^{2}}-\frac{\beta_{T}^{2} T}{\rho c_{p}}\right]
$$

For pure substances such as ideal gases $\left(\beta_{T} \approx 1 / T\right)$ and liquids like water $\left(\gamma \approx 1, \beta_{T} \approx 0\right)$ the classical approximation $\frac{1}{\rho c_{\text {eff }}^{2}} \approx \frac{1}{\rho c^{2}}$ is justified if we neglect viscous dissipation. In problems involving multiple phases, we average Eq. 10 implying that the mixture compressibility is given by the harmonic mean of the compressibilities

$$
\frac{1}{\rho c_{\mathrm{eff}}^{2}}=\frac{C}{\rho_{1} c_{\mathrm{eff}, 1}^{2}}+\frac{1-C}{\rho_{2} c_{\mathrm{eff}, 2}^{2}} .
$$

The system of equations solved is closed by adding an equation of state (EOS) for each phase that establishes the relation between the various thermodynamic variables $\operatorname{EOS}_{\mathrm{i}}\left(\mathrm{p}_{\mathrm{i}}, \mathrm{T}_{\mathrm{i}}, \rho_{\mathrm{i}}\right)=0$. Note that from the state equation we can obtain various quantities such as the speed of sound, the thermal dilatation coefficient, etc. In this work we use the general EOS written in the Mie-Gruneisen form (see for example [25, 26])

$$
\rho_{i} e_{i}=\frac{p_{i}+\Gamma_{i} \Pi_{i}}{\Gamma_{i}-1}
$$


which defines the sound speed as

$$
c_{i}^{2}=\Gamma_{i} \frac{p_{i}+\Pi_{i}}{\rho_{i}},
$$

but in principle any other choice of the EOS is possible.

\section{Numerical scheme}

In this section we present the numerical scheme used to solve the equations detailed in the previous section using a cell centered formulation for octree grids [19].

For a two-component mixture, we obtain the evolution of the interface position (Eq. 1) using the Volume-Of-Fluid (VOF) method that solves the advection equation for the color function $C$ representing the volume of the reference phase with respect to the total volume of the grid. This equation is solved numerically following the split advection method proposed by Weymouth \& Yue [27] where the advection equation for the volume fraction is written as

$$
\frac{\partial C}{\partial t}+\nabla \cdot(\boldsymbol{u} C)=C \nabla \cdot \boldsymbol{u} .
$$

This form of the equation is valid for both compressible and incompressible substances and has been used in previous schemes based on Riemann solvers for multiphase flow simulations [26]. In a direction-split advection method the discretized advection equation for $C$ takes the form

$$
C^{n+1}=C^{n}-\sum_{f} F_{C}\left(u_{f} \Delta t / \Delta x, C\right)+C^{*} \sum_{f} \nabla_{f} \boldsymbol{u} \Delta t
$$

where $f$ denotes the cell face, $F_{C}=C_{\text {adv }} u_{f}$ is related to the flux of $C$ across the face during $\Delta t$, and $C_{\mathrm{adv}}$ is the fraction of the reference phase crossing the face during $\Delta t$ evaluated geometrically. The right-hand-side of the equation represents the volume change of the reference phase during $\Delta t$ due to compressibility effects and as explained in [27], $C^{*}$ must be held constant for all sweep directions in order to ensure exact volume (e.g. mass) conservation in the incompressible limit. 
In the time-split scheme the set of conservative equations is solved in various steps. The advection step solves for the advection of mass, momentum and energy in each phase separately to obtain provisional values of the conservative quantities associated with the $i$ th component as

$$
\left(C_{i} \boldsymbol{Y}_{i}\right)^{*}=\left(C_{i} \boldsymbol{Y}_{i}\right)^{n}-\sum_{f} \boldsymbol{F}_{C_{i} \boldsymbol{Y}_{i}}\left(\Delta t u_{f} / \Delta x, C_{i}, \boldsymbol{Y}_{i}\right)
$$

where $\boldsymbol{Y}_{i}=\left(\rho_{i}, \rho_{i} \boldsymbol{u}, \rho_{i} e_{i}+1 / 2 \rho_{i}|\boldsymbol{u}|^{2}\right)^{T}$ and $\boldsymbol{F}_{\boldsymbol{Y}_{i}}$ represents the amount of mass, momentum or energy transfered across a given computational face during the time step. Because the transport of the color function $C$ and the conservative quantity $C_{i} \boldsymbol{Y}_{i}$ are obviously linked, the calculation of the flux terms is expressed as a function of the flux of $C$ obtained geometrically to solve Eq. 15

$$
\boldsymbol{F}_{C_{i} \boldsymbol{Y}_{i}}\left(\Delta t u_{f} / \Delta x, C_{i}, \boldsymbol{Y}_{i}\right)=F_{C}\left(u_{f} \Delta t / \Delta x, C_{i}\right) \boldsymbol{Y}_{i, a d v},
$$

where $\boldsymbol{Y}_{i, a d v}$ represents the averaged value of the conservative quantity related to the $i$ th component within the advected volume crossing the face during the timestep. It is important to remark that by recycling the value of $F_{C}$ we can freely chose the method to obtain $\boldsymbol{Y}_{i, a d v}$ while minimizing the error coming from inconsistencies between the advection of the color function and the conservative quantity $C_{i} \boldsymbol{Y}_{i}$ in mixed cells during the overall timestep. To see this, let's consider a problem where we know the amount of $c$ crossing a cell face during a given $\Delta t$

$$
F_{c_{1}}=\int_{f} c_{1}(t) u_{f}(t) d t
$$

In this case the corresponding flux of $c_{1} y_{1}$

$$
F_{y_{1} c_{1}}=\int_{f} y_{1}(t) c_{1}(t) u_{f}(t) d t
$$

could be computed using an approximation of the full integral $g(t)=y_{1}(t) c_{1}(t) u_{f}(t)$ by the Taylor expansion

$$
g(t)=g\left(t_{0}\right)+\left.\frac{\partial g}{\partial t}\right|_{t_{0}}\left(t-t_{0}\right)+\left.\frac{1}{2} \frac{\partial^{2} g}{\partial t^{2}}\right|_{t_{0}}\left(t-t_{0}\right)^{2}+\mathcal{O}\left(\Delta t^{3}\right) .
$$

However this approximation does not converge when, as it is the case, $g(t)$ is a discontinuous function. The problem can be solved if we define a smooth 
approximation of $y_{1}$ at the $\mathrm{n} t h$ order as $y_{1} \approx \overline{y_{1}}+\mathcal{O}\left(\Delta t^{n}\right)$. In this case we can rewrite Eq. 19 as

$$
F_{y_{1} c_{1}}=F_{c_{1}}\left(\overline{y_{1}}+\mathcal{O}\left(\Delta t^{n}\right)\right)
$$

which is consistent with the calculation of the flux of $C_{i}$ obtained from Eq. 18 at the order of the approximation used to estimate $y_{i}$. Following this procedure, the problem of the transport of a discontinuous function depending on $C$ is reduced to the problem of obtaining the volume fraction flux (Eq. 18) naturally handled by the VOF method. For practical purposes we evaluate $\boldsymbol{Y}_{i, a d v}$ using the Bell-Collela-Glaz second-order upwind scheme [28],

$$
\boldsymbol{Y}_{i, a d v}= \begin{cases}\boldsymbol{Y}_{i}+\frac{1}{2} \operatorname{sign}\left(\mathrm{u}_{\mathrm{f}}\right) \min \left(1,1-\operatorname{sign}\left(\mathrm{u}_{\mathrm{f}}\right) \frac{\mathrm{u}_{\mathrm{f}} \Delta \mathrm{t}}{\Delta \mathrm{x}}\right) \frac{\partial \boldsymbol{Y}_{\mathrm{i}}}{\partial \mathrm{x}} \Delta \mathrm{x}, & \text { if } C_{i}>0 \\ 0, & \text { otherwise }\end{cases}
$$

where the values of $C_{i}$ and $\boldsymbol{Y}_{i}$ are upwind cell centered values. The scheme is adapted to define a smooth function of $\boldsymbol{Y}_{i, a d v}$ in regions close to the interface by obtaining the derivative of $\boldsymbol{Y}_{i}$ in the jth cell as

$$
\left.\frac{\partial \boldsymbol{Y}_{i}}{\partial x}\right|^{j}= \begin{cases}\frac{1}{\Delta x} f_{L}\left(\boldsymbol{Y}_{i}^{k-1}, \boldsymbol{Y}_{i}^{k}, \boldsymbol{Y}_{i}^{k+1}\right), & \text { if } C_{i}^{k}>0 k=j-1, j, j+1 \\ \frac{\boldsymbol{Y}_{i}^{k}-\boldsymbol{Y}_{i}^{k-1}}{\Delta x}, & \text { if } C_{i}^{k+1}=0 \wedge C_{i}^{k-1}>0 \\ \frac{\boldsymbol{Y}_{i}^{k+1}-\boldsymbol{Y}_{i}^{k}}{\Delta x}, & \text { if } C_{i}^{k-1}=0 \wedge C_{i}^{k+1}>0 \\ 0, & \text { otherwise, }\end{cases}
$$

where $f_{L}\left(\boldsymbol{Y}_{i}^{k-1}, \boldsymbol{Y}_{i}^{k}, \boldsymbol{Y}_{i}^{k+1}\right)$ is a function that provides an estimation of the increment of a variable in a given direction. In this work we use the generalized minmod slope limiter

$$
f_{L}\left(y_{-1}, y_{0}, y_{1}\right)= \begin{cases}\min \left(\theta\left(y_{0}-y_{-1}\right), \frac{y_{1}-y_{-1}}{2}, \theta\left(y_{1}-y_{0}\right)\right), & \text { if } y_{-1}<y_{0}<y_{1} \\ \max \left(\theta\left(y_{0}-y_{-1}\right), \frac{y_{1}-y_{-1}}{2}, \theta\left(y_{1}-y_{0}\right)\right), & \text { if } y_{-1}>y_{0}>y_{1} \\ 0, & \text { otherwise }\end{cases}
$$

where $\theta$ is a freely adjustable parameter that allows to recover various classical slope limiters such as superbee $(\theta=2)$ and minmod $(\theta=1)$. Note that we use slope limiting rather than flux limiting. The numerical calculations included in this manuscript show a negligible dependence on the parameter $\theta$. 
The advection scheme proposed presents some interesting properties. First, by construction, the advection step is fully conservative in the discrete framework. Second, the fluxes obtained for the color function and the conservative quantities are consistent, significantly reducing errors appearing during the advection of a discontinuity. Finally, because the advection step is taken for each phase separately, we avoid any artificial numerical diffusion between the two immiscible phases. This is important in the case of large density differences since methods ensuring the conservation of momentum and energy are known to reduce the appearance of numerical instabilities in the simulation of multiphase incompressible flow problems [29, 30, 31].

After the advection step the provisional momentum is corrected and viscosity or surface tension effects are taken into account as

$$
\begin{aligned}
\frac{(\rho \boldsymbol{u})^{* *}-(\rho \boldsymbol{u})^{*}}{\Delta t} & =\nabla \cdot\left(\mu\left(\nabla \boldsymbol{u}+(\nabla \boldsymbol{u})^{T}\right)\right)-\frac{2}{3} \nabla \cdot(\mu(\nabla \cdot \boldsymbol{u}) \boldsymbol{I}), \\
\frac{(\rho \boldsymbol{u})^{* * *}-(\rho \boldsymbol{u})^{* *}}{\Delta t} & =\sigma \kappa \nabla C .
\end{aligned}
$$

The calculation of the surface tension forces is critical for accurate results. This step is similar to that already described in Popinet $[18,11]$ and therefore the reader is referred to these publications for further details.

Finally, in the classical projection step we take the divergence of the remaining terms in the momentum equation

$$
\boldsymbol{u}^{n+1}-\boldsymbol{u}^{* * *}=-\frac{\Delta t}{\rho} \nabla p
$$

The divergence of the velocity at the end of the time step is expressed as a function of pressure by splitting Eq. 10 in time as

$$
\begin{array}{r}
\frac{p^{*}-p^{n}}{\Delta t}+\boldsymbol{u} \cdot \nabla p=0 \\
\frac{1}{\rho c_{e}^{2}} \frac{p^{n+1}-p^{*}}{\Delta t}=-\nabla \cdot \boldsymbol{u}^{n+1}+\frac{\beta_{T} \Phi_{v}}{\rho c_{p}} .
\end{array}
$$

Replacing Eq. 29 into Eq. 27 we find the following Poisson-Helmhotz equation for pressure

$$
\frac{1}{\rho c_{\mathrm{eff}}^{2}} \frac{p^{n+1}-p^{*}}{\Delta t}+\nabla \cdot \boldsymbol{u}^{* * *}-\frac{\beta_{T} \Phi_{v}}{\rho c_{p}}=\nabla \cdot\left(\frac{\Delta t}{\rho} \nabla p\right),
$$


where the divergence terms are computed by linear interpolation of the cell centered values for $\boldsymbol{u}^{* * *}$, which defines a provisional staggered velocity field. For the calculations reported in this manuscript we use

$$
\frac{1}{\rho c_{\mathrm{eff}}^{2}} \approx \frac{\overline{\frac{1}{\gamma-1}}}{p\left(\overline{\frac{1}{\gamma-1}}+1\right)+\overline{\frac{\Pi \gamma}{\gamma-1}}}
$$

where

$$
\begin{array}{r}
\frac{1}{\gamma-1}=\frac{C}{\gamma_{1}-1}+\frac{1-C}{\gamma_{2}-1} \\
\frac{\Pi \gamma}{\gamma-1}=C \frac{\Pi_{1} \gamma_{1}}{\gamma_{1}-1}+(1 .-C) \frac{\Pi_{2} \gamma_{2}}{\gamma_{2}-1}
\end{array}
$$

The method followed to solve for Eq. 28 to obtain $p^{*}$ significantly influences the performance of the method. Among the various alternatives proposed in the literature $[13,14,15,16]$ we chose the solution proposed by Xiao et al. [15] (also used in [16]) where pressure is computed from the total energy obtained after the advection step. Thus, although Eq. 10 is sufficient to close the system of equations because it implicitly contains the energy equation, we explicitly solve the conservative form of the energy equation in order to compute the provisional pressure $p^{*}$ as

$$
p^{*}=\frac{(\rho E)^{*}-\frac{1}{2} \rho|\boldsymbol{u}|^{2}-\frac{\overline{\Pi \gamma}}{\gamma-1}}{\frac{1}{\gamma-1}},
$$

where $(\rho E)^{*}=c \rho_{1} e_{1}+(1-c) \rho_{2} e_{2}+\frac{1}{2} \rho|\boldsymbol{u}|^{2}$ is the total energy computed with the provisional values after the advection step. This step guarantees that the energy is conserved at the discrete level in the absence of viscous dissipation and surface tension.

Once the pressure at the end of the timestep has been obtained we update $\boldsymbol{u}$ at the faces using Eq. 27 and the total momentum defined at the cell center as

$$
(\rho \boldsymbol{u})^{n+1}-(\rho \boldsymbol{u})^{* *}=-\Delta t \nabla^{c} p,
$$

where $\nabla^{c} p$ is obtained as a simple average between the face values of the pressure gradient. Finally, the total energy for each phase is updated as

$$
\left(\rho_{i} e_{i}+1 / 2 \rho_{i} \boldsymbol{u}^{2}\right)^{n+1}=\left(\rho_{i} e_{i}+1 / 2 \rho_{i} \boldsymbol{u}^{2}\right)^{*}+\Delta t\left(-\nabla \cdot\left(\boldsymbol{u} p_{i}\right)+\nabla \cdot\left(\tau_{i}^{\prime} \boldsymbol{u}\right)\right) .
$$


For the calculation of the divergence terms we use the face velocities already obtained and the pressure of the ith component at the face. For faces in contact with two single-component cells we define the pressure at the face as the average between the two neighboring cells. When the interface is present we account for the fact that Eq. 30 is indeed solved for the averaged pressure $p=C p_{1}+(1-C) p_{2}$, from which we compute the pressure of the ith face using the Laplace relation

$$
\begin{aligned}
& p_{1}=p+(1-C) \sigma \kappa \\
& p_{2}=p-C \sigma \kappa .
\end{aligned}
$$

The face pressure is finally taken as the cell-centered pressure of the cell that contains the largest volume fraction between the two neighboring cells. The full description of the algorithm is resumed in the flow chart 1.

\section{Numerical results}

\subsection{Single phase problems}

\subsubsection{Propagation of a linear pressure disturbance in a fluid}

In order to investigate the numerical properties of the method in the linear regime we measure the numerical dispersion of a small, one-dimensional pressure disturbance in an inviscid fluid. The initial condition is given by the pressure field $p(x, t=0)=p_{0}\left[1+2 \Delta p \exp \left(-4 x^{2} / \chi^{2}\right)\right]$ in a fluid at rest (zero velocity) where the domain of size $L_{0}=10$ is discretized with a regular cartesian grid of size $\Delta x / L_{0}=1 / 2^{10}$. The liquid density and speed of sound is one and the pressure perturbation is obtained using $\Delta p=5 \times 10^{-3}$ and $\chi=0.1$.

In the acoustic limit, $\Delta p \ll 1$, nonlinear terms are negligible and the advection step does not impact the results. The pressure pulse splits and generates two waves propagating in opposite directions. In the absence of any physical dissipation the frequency content remains unchanged as the wave propagates at the reference sound speed $c_{0}$. We obtain the spatial distribution of pressure at four different instants $\left(\frac{c_{0} t}{\chi}=4,12,20,28\right)$ and we perform a Fast Fourier Transform (FFT) to decompose the signal into spatial Fourier modes. For each wavelength we fit the decay rate in time of the absolute module of the FFT to an exponential decay of the type $C_{0} e^{-A t}$, 


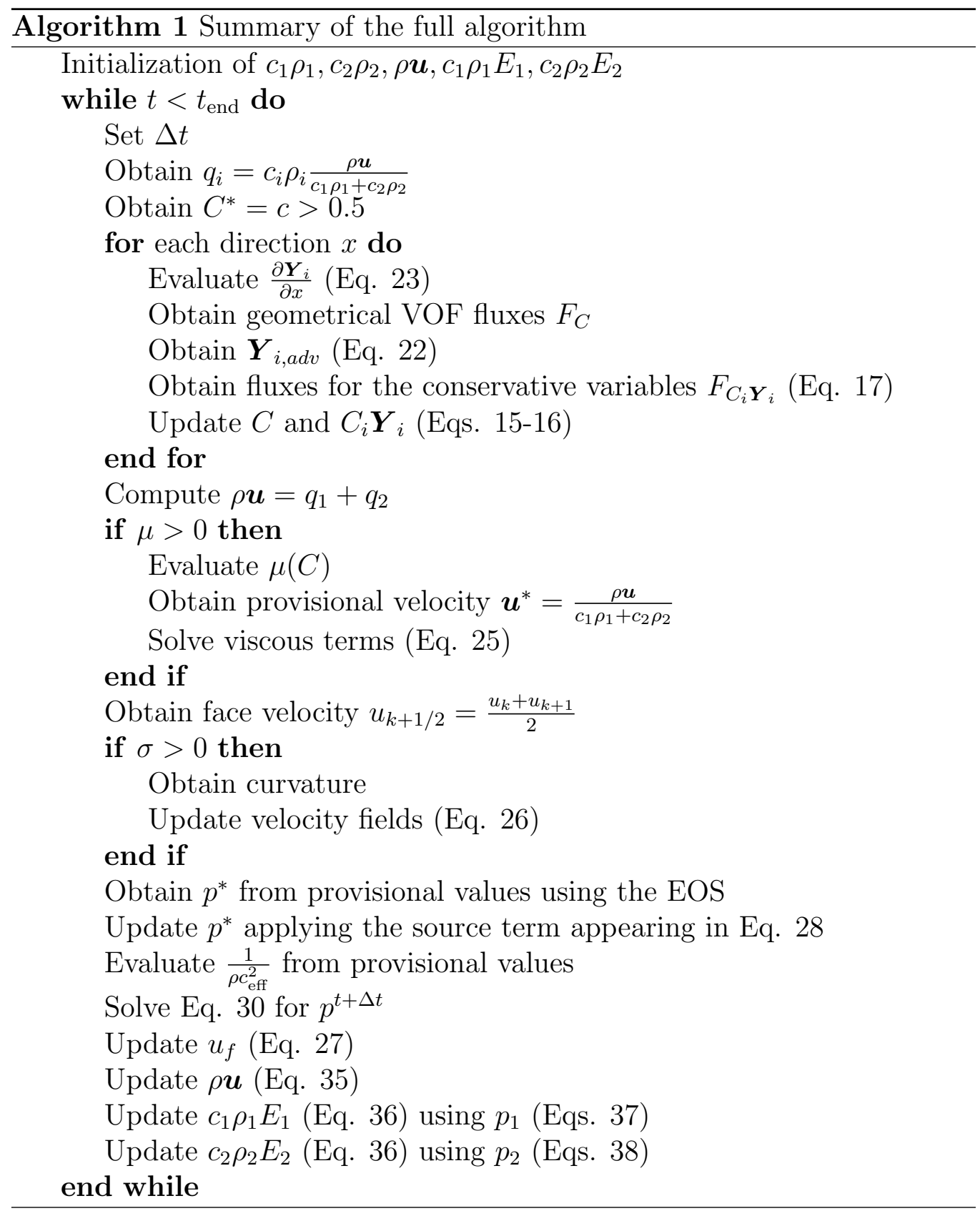


where $A$ is directly related to the numerical dissipation at a given length scale.
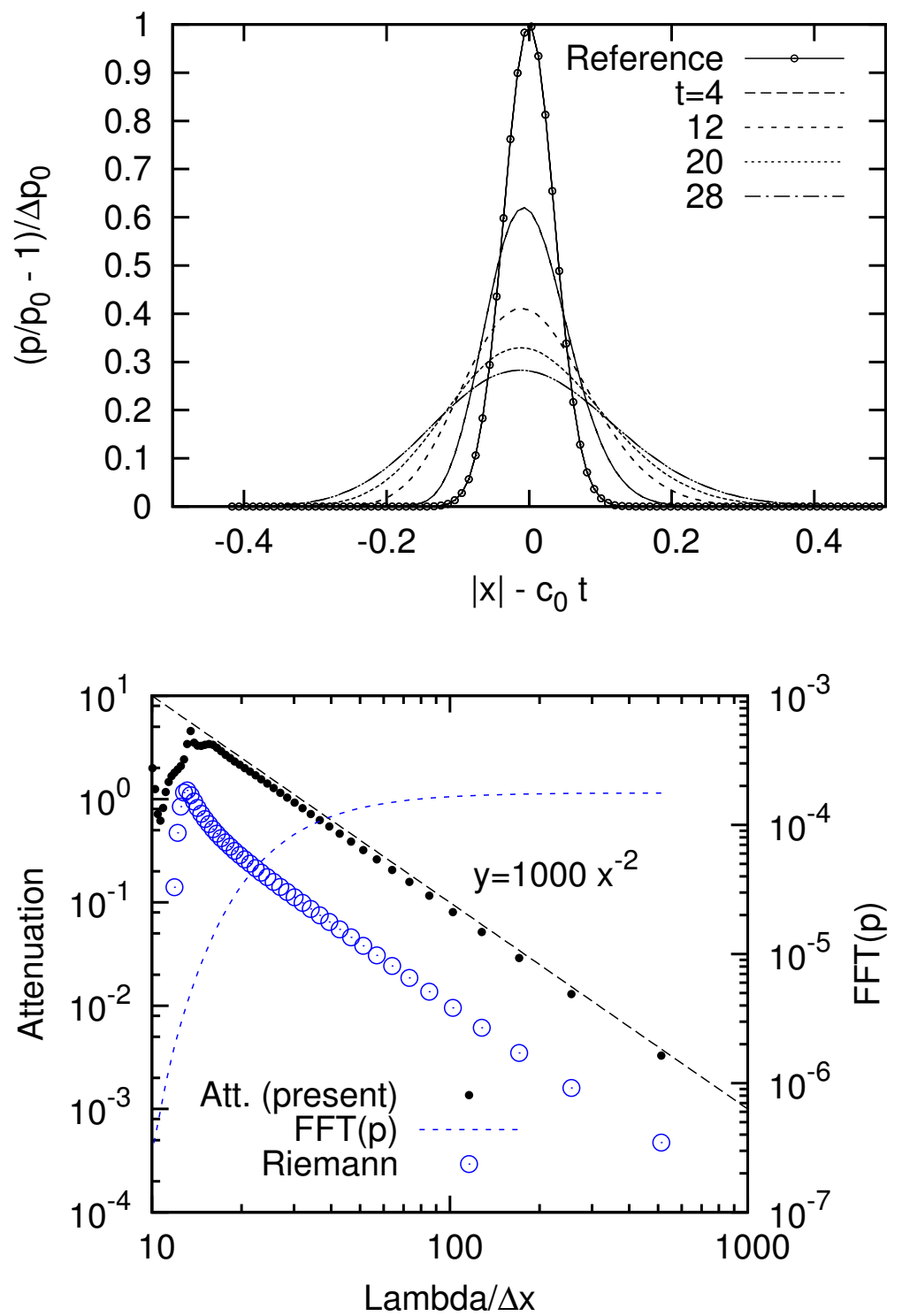

Figure 1: Top: Evolution of the pressure pulse shape as a function of time for CFL=0.5. Bottom: Effective attenuation induced by the numerical scheme as a function of the wavelength. 
Figure 1 shows the results obtained with $\mathrm{CFL}_{\mathrm{ac}}=\frac{\mathrm{c}_{0} \Delta \mathrm{t}}{\Delta \mathrm{x}}=0.5$. The pulse peak propagates at the correct speed but it is dissipated as a consequence of the properties of the numerical method. The measurement of the effective attenuation as a function of wavelength, which is a measure of the discretization error, converges to second order. It is important to remark that the numerical dissipation of the current method is larger than that measured using a classical second-order Riemann solver. To obtain comparable results in terms of accuracy between the two methods, one needs to double the resolution of the all-Mach formulation.

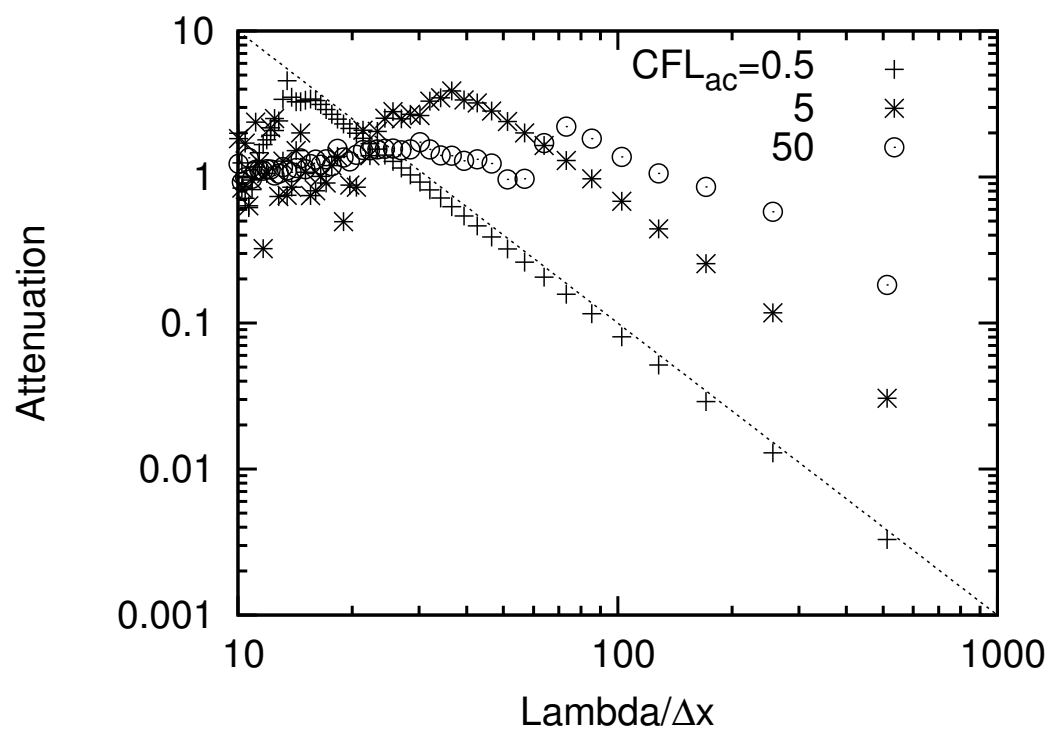

Figure 2: Influence of the CFL number on the effective attenuation. The method is shown to converge with the time-step (CFL) at first order. The cut-off distance below which the numerical dissipation saturates also depends linearly on the CFL.

Figure 2 shows the impact of the CFL number. As we can see the method allows for stable solutions for $\mathrm{CFL}_{\mathrm{ac}}$ larger than 0.5 at the expense of increasing the effective attenuation of the wave and increasing the cut-off distance beyond which attenuation saturates. Defining the critical scale $\lambda_{c}$ as the minimum length beyond which we do no longer observe convergence, we find that $\frac{\lambda_{c}}{\Delta x}=40 \times \mathrm{CFL}_{\mathrm{ac}}$, which can be also written as $\frac{\lambda_{c}}{c_{0} \Delta t}=40$. We can interpret this as the fact that the proposed scheme neglects compressibility 
effects for wavelengths $\lambda<\lambda_{c}$, with $\lambda_{c}$ tending to infinity as we approach the incompressible limit.

\subsubsection{Propagation of a shock wave in an ideal gas}

In this problem we solve for a 1D shock wave propagating in an ideal gas with $\gamma=1.4$ in order to test the scheme in a situation where nonlinear advection terms are relevant. The Rankine-Hugoniot conditions are used to initialize the solution defining the right (pre-shocked) and left (shocked) state as $p_{L}=10, p_{R}=0.1, \rho_{R}=1$. The theoretical shock speed is

$$
\frac{s}{c_{0}}=\sqrt{\frac{\frac{\gamma+1}{2}}{\gamma\left(\frac{p_{L}}{p_{R}}-1\right)+1}},
$$

where $c_{0}=\sqrt{\frac{\gamma p_{R}}{\rho_{R}}}$.

Figure 3 shows the shock wave structure in the frame of reference of the theoretical front solution, $x_{\text {front }}=s t$. The method is shown to be able to capture the correct shock speed. The thickness of the shockwave is approximately three cells. It should be noted that the numerical oscillation behaves in a way different from the conventional density-based shock capturing schemes. The upwind method minimizes the spurious oscillations at the expense of a larger error for a given resolution relative to the BCG 2ndorder advection schemes, which shows an almost negligible influence of the parameter $\theta$ on the solution. Remarkably the overall error computed based on the $L_{1}$ norm is indeed smaller in the case of the BCG advection scheme and first-order convergence is obtained for all cases as expected for a problem including shocks.

\subsection{Two phase problems}

\subsubsection{Isolated interface problem}

In this section we test the full solver for the problem of advection of an interface between two different ideal gases at uniform velocity and pressure. This test proposed in [26] quantifies the amplitude of spurious pressure and velocity oscillations induced by the method when advecting an interface with different material properties. The interface is initially placed at the middle of 

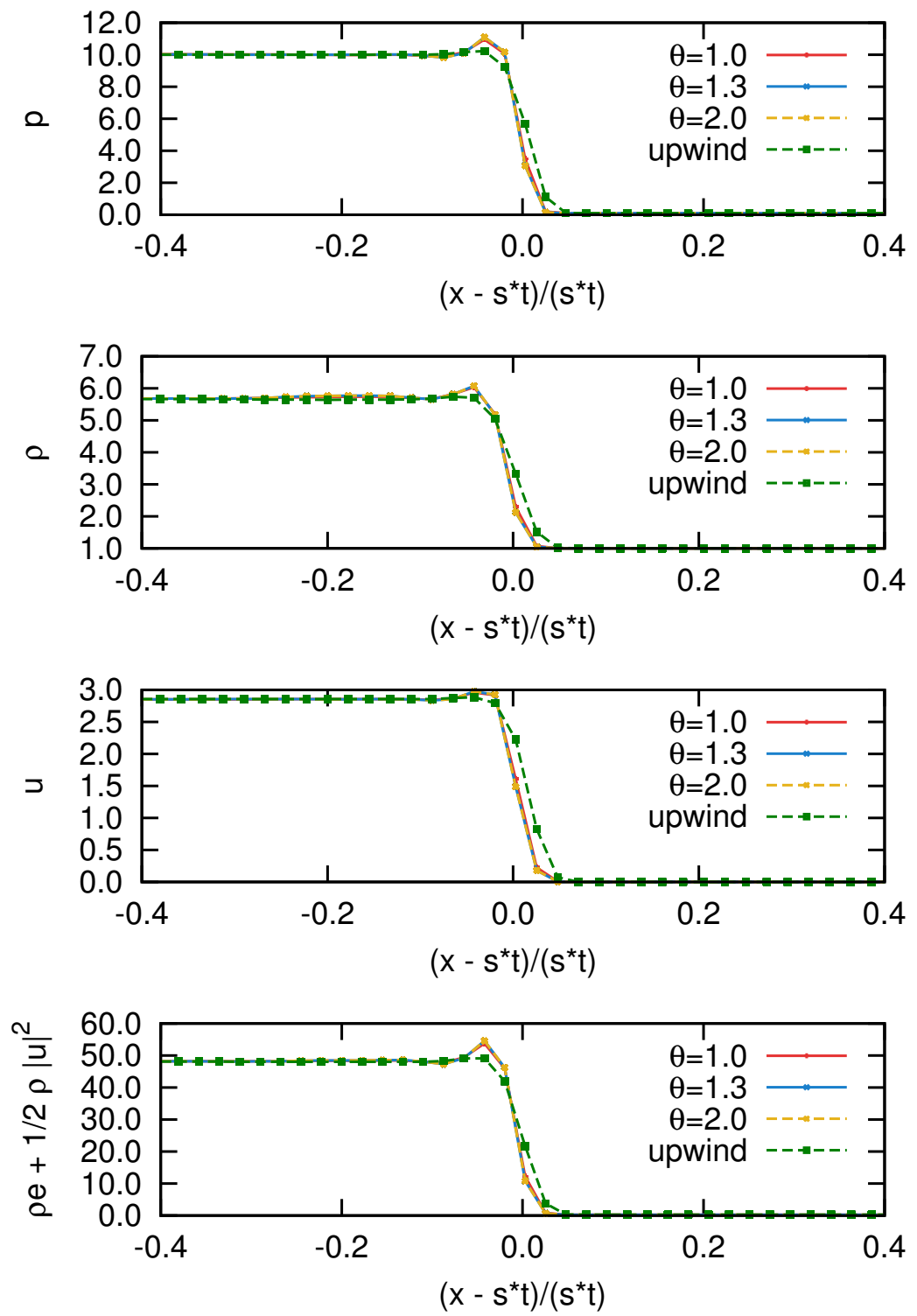

Figure 3: Structure of the shockwave at $\mathrm{t}=1$ for the Bell-Colella-Glaz (BCG) advection scheme and a simple 1st-order upwind method.

the domain of length $L_{0}=2$ discretized with 128 cells per direction where periodic boundary conditions are applied. The left and right states are defined 

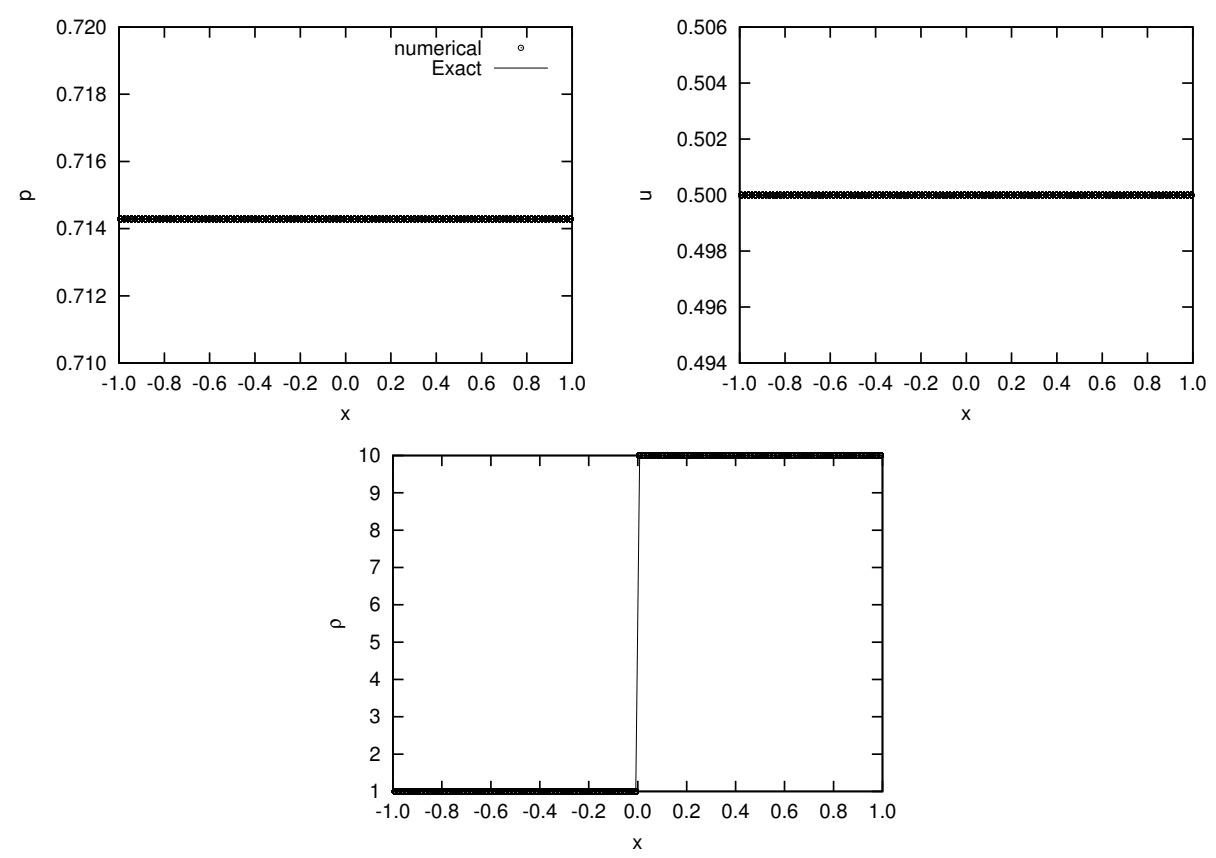

Figure 4: Pressure, velocity and density profiles after the advection of an isolated interface between two ideal gases at uniform pressure and velocity.

as

$$
\begin{gathered}
(\rho, u, p, \gamma)_{L}^{T}=(1,0.5,1 / 1.4,1.2)^{T} \\
(\rho, u, p, \gamma)_{R}^{T}=(10,0.5,1 / 1.4,1.4)^{T}
\end{gathered}
$$

By construction the method is designed to consistently transport the color function and all the conservative quantities during the advection step leading to a uniform provisional pressure and velocity that does not need to be corrected during the projection step. The results summarised in Figure 4 demonstrate that the present method is able to advect the interface between the two fluids at the theoretical speed without inducing any significant oscillation on the pressure and velocity fields keeping the interface sharp. Discrete conservation is also observed within round-off error. 


\subsubsection{Spurious currents in a static configuration}

One common test for multiphase incompressible methods dealing with surface tension is the measurement of the parasitic currents induced by the discretization errors on the initialization $[18,11]$. In this section we report the results obtained for a two-dimensional bubble of radius $R=0.4$ containing an ideal gas $\left(\Gamma_{g}=\gamma_{g}=1.4, \Pi_{g}=0\right)$ in a slightly compressible liquid $\left(\Gamma_{l}=7.14, \Pi_{l}=300\right)$. The ambient liquid pressure is $p_{0}=1$ and the liquid and gas density is set to an initial constant value $\rho_{l, 0}=\rho_{b, 0}=1$. The initial gas pressure is given by the Laplace pressure $p_{g, 0}=p_{0}+\frac{\sigma}{R_{0}}$, where the surface tension is set to $\sigma=1$. We test various values of the fluid viscosity (equal for both substances) in order to modify the Laplace number $\mathrm{La}=\frac{\rho \mathrm{D}_{0} \sigma}{\mu^{2}}$ that controls the dissipation rate of the fluctuations induced by the initial discretization error. The grid size is kept constant and equal to $R / \Delta x=12.8$.

As shown in Figure 5, the method reduces the amplitude of the spurious currents on a time scale proportional to the viscous scale, $t_{\text {visc }} \propto D^{2} / \mu$ for all the Laplace numbers tested here. Comparing the decaying rate of the fluctuations with those obtained in [18] in the incompressible limit, significant differences are only observed for very large Laplace numbers where the incompressible version tends to dissipate the spurious currents faster than the current version of the solver including compressibility effects. Note that at large Laplace numbers the viscous boundary layer is most likely not captured by the grid resolution and therefore the observed dissipation rate is mainly controlled by numerical scheme properties which are different in the incompressible and compressible framework. In particular the incompressible formulation does not explicitly include any form of energy equation whereas in the compressible formulation the total energy dissipation is directly controlled by the explicit calculation of the viscous dissipation and the discretization errors on the representation of the surface energy included through the pressure jump across the interface.

Another important difference between the compressible and incompressible formulation is the appearance of high frequency fluctuations that occur in a time scale much shorter than the viscous time scale. Figure 6 reveals that indeed these fluctuations occur due to the resonance properties of the gas bubble whose characteristic time is $t_{\text {res }}=\frac{1}{f_{0}} \propto R_{0} \sqrt{\frac{\rho_{l}}{\gamma_{g} p_{0}}}$. We can then conclude that the discretization errors on the surface tension acts as an ini- 


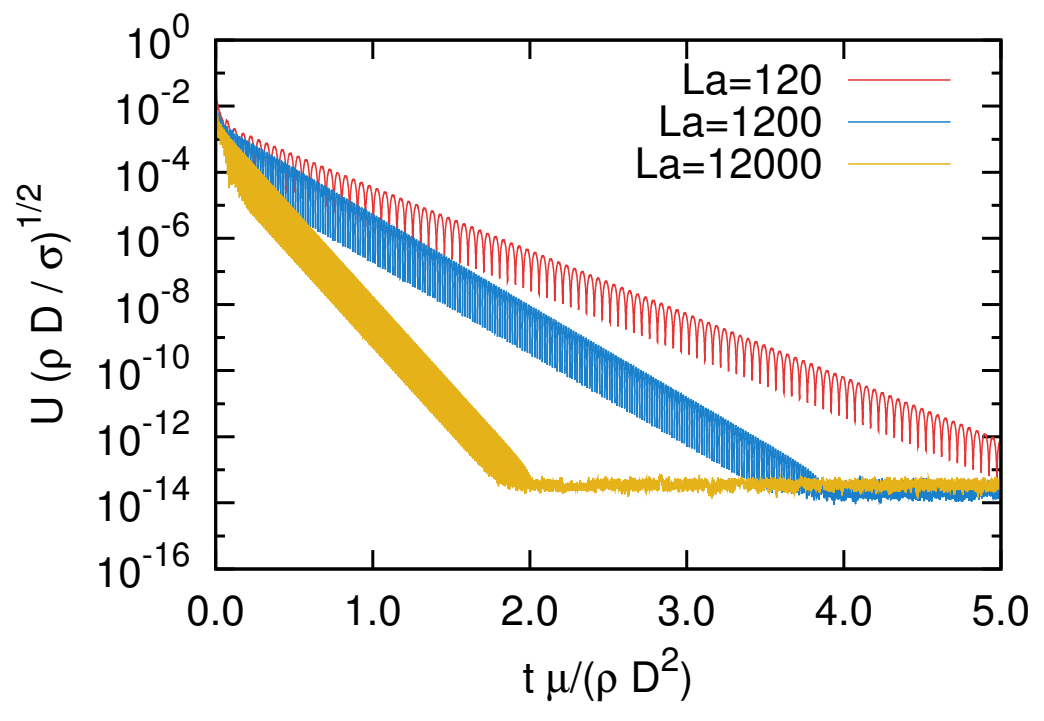

(a)

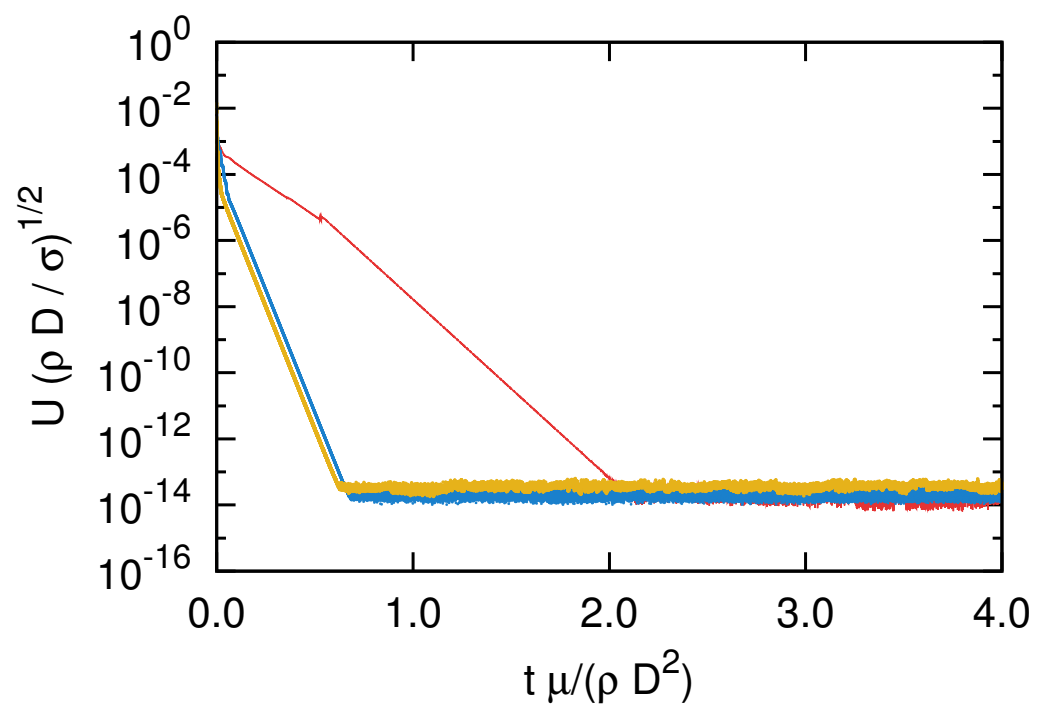

(b)

Figure 5: Evolution of the velocity fluctuations of a 2D bubble as a function of the Laplace number using (a) the nondimensional viscous time for the all-Mach solver and (b) the incompressible solver of [18]. 


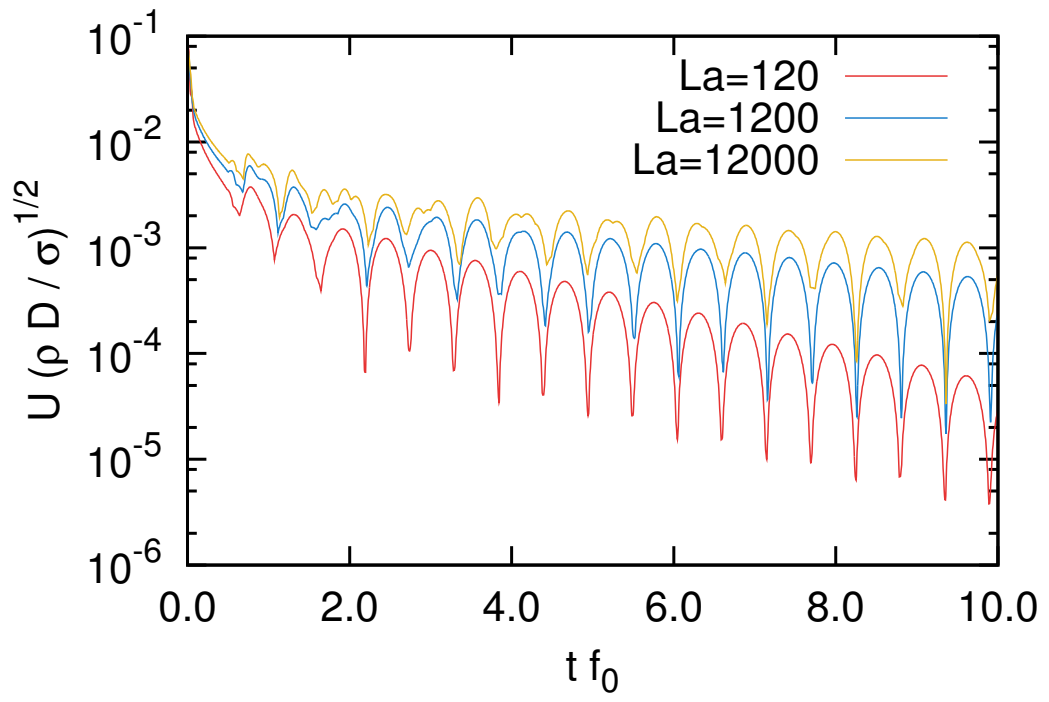

Figure 6: Evolution of the velocity fluctuations as a function of the Laplace number using a nondimensional time defined with the bubble resonance frequency.

tial impulsive force that makes the bubble oscillate mainly at its resonance frequency.

\subsubsection{Linear oscillation of a single bubble}

In this section we investigate the three-dimensional problem of the linear bubble oscillation induced by a sinusoidal acoustic wave of given frequency $\omega$ where surface tension and viscous effects are neglected. Properties are made dimensionless using the reference pressure and liquid density, $p_{0}=1, \rho_{l, 0}=1$. We initialize a gas bubble $\left(\Gamma_{g}=\gamma_{g}=1.4 \Pi_{g}=0\right)$ of initial radius $R_{0}=1$ and density $\rho_{g, 0} / \rho_{l, 0}=10^{-3}$ at the center of a cubic domain of length $L_{0}=3 \lambda$, where $\lambda=c_{0} / f$ is the wavelength of the wave induced in the system. The EOS of the liquid is defined by $\Gamma_{l}=7.14$ and $\frac{\Pi_{l}}{p_{0}}=3000$ and the initial velocity field $\boldsymbol{u}=\left(U_{0}, 0,0\right)$ is given by

$$
u(x, 0)= \begin{cases}U_{0} \sin (k(x+2)) & -\lambda<x+2<0 \\ 0 & \text { otherwise }\end{cases}
$$


where $U_{0}=10^{-3}$ and $k=\frac{\omega}{c_{0}}$ is the wavenumber that will be varied. The initial pressure field is given by the linear approximation $p(x, 0)=p_{0}+$ $\rho_{l, 0} c_{0} u(x)$. Each simulation is run for $t f=1$. An adaptive grid is used to refine the region near the bubble but grid is progressively coarsened far from the domain center.

In the linear regime and in absence of viscous and surface tension effects the bubble response solely depends on the ratio between the excitation frequency, $\omega$, and the bubble resonance frequency, $\omega_{\text {res }}=\frac{1}{R_{0}} \sqrt{\frac{3 p_{0} \gamma_{g}}{\rho_{l}}}=\sqrt{4.2}$. Because in the linear regime non-spherical bubble deformation effects are high order corrections of the exact solution and therefore negligible, the evolution of the averaged bubble radius obtained from the solution of the RayleighPlesset (RP) equation is expected to provide an accurate estimation of the exact solution

$$
\ddot{R} R+\frac{3}{2} \dot{R}=\frac{1}{\rho_{l}}\left(p_{b, 0}\left(\frac{R_{0}}{R}\right)^{3 \gamma}-4 \mu_{l} \frac{\dot{R}}{R}-p_{\infty}(t)\right),
$$

where $p_{\infty}(t)=p_{0}+\rho_{l} c_{0} U_{0} \sin (\omega t)$.

Figure 7 shows the temporal evolution of the bubble volume $V$ as a function of the grid size for an excitation frequency above and below the bubble resonance frequency, $\frac{\omega}{\omega_{\text {res }}}=2$, and $\frac{\omega}{\omega_{\text {res }}}=0.1$ respectively. For frequencies above the bubble natural frequency the method converges accurately to the solution predicted by the Rayleigh-Plesset model. For frequencies much below the resonance frequency the problem becomes challenging due to the very different scales between the wavelength and the bubble radius. We observe the appearance of fluctuations of small amplitude at the bubble resonance frequency during the initial transient state that are only partially captured due to the limited resolution of the full three-dimensional simulation and the sensitivity of these fluctuations to the characteristics of the incoming pressure pulse. These transient, high-frequency fluctuations are indeed damped by adding a small amount of viscosity to the liquid in the Rayleigh-Plesset model. From the results of Figure 7 we can then conclude that coarsening the grid increases the effective damping of the solution obtained which is an expected behavior of the numerical scheme when the resolution is low. In any case the agreement between numerical and theoretical solutions is satisfactory both, above and below the bubble resonance frequency. 

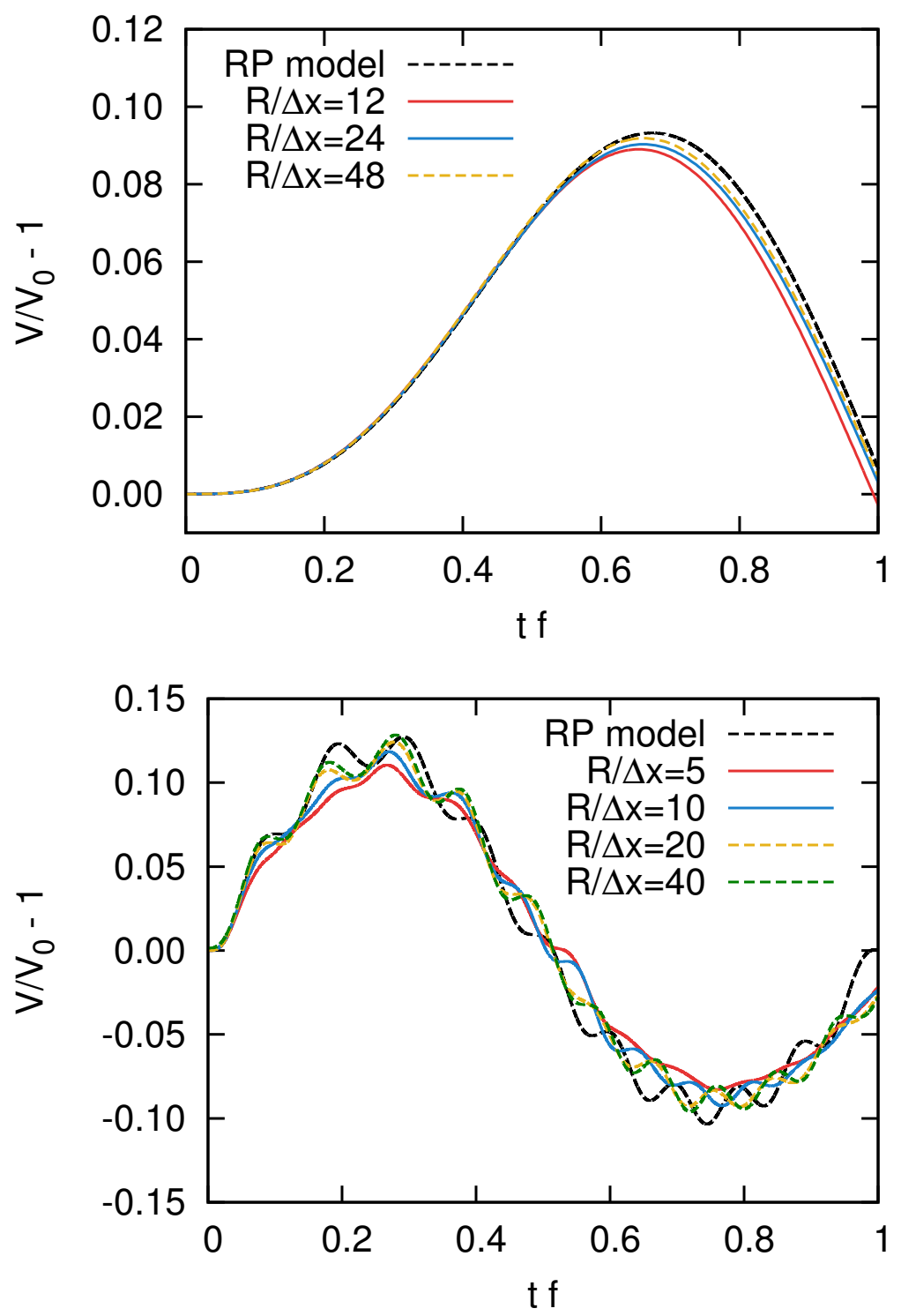

Figure 7: Top: Temporal evolution of the bubble volume for $\omega / \omega_{\text {res }}=2$ as a function of the grid resolution. For comparison, the solution obtained with a Rayleigh-Plesset (RP) equation is included. Bottom: Solution obtained for $\omega / \omega_{\text {res }}=0.1$ (right). As reference we include the solution of the RP model. 


\begin{tabular}{cccccc}
$p_{l, 0} / p_{b, 0}$ & $r$ & $m$ & $\mathrm{Re}$ & $\mathrm{We}$ & $\mathrm{Ma}$ \\
\hline 5 & $10^{-3}$ & $10^{-2}$ & 10 & 400 & $4.3 \times 10^{-2}$
\end{tabular}

Table 1: Non-dimensional parameters for the collapse of a bubble in a viscous liquid used in Section 4.2.4.

\subsubsection{Weakly non-linear collapse of a single bubble in a viscous fluid}

In order to test nonlinear terms in a multiphase flow problem, we consider the so-called Rayleigh collapse problem [32] where a single bubble with initial pressure $p_{b, 0}$ is suddenly exposed to a pressure increase in the surrounding liquid $\frac{p_{l, 0}}{p_{b, 0}}>1$. An initially spherical gas bubble $\left(\Gamma_{g}=\gamma_{g}=1.4, \Pi_{g}=0\right)$ of radius $R_{0}=1$ at uniform pressure $p_{b, 0}=100$ and density $r=\frac{\rho_{b, 0}}{\rho_{l, 0}}$, collapses in a liquid with density $\rho_{l, 0}=1$ where the parameters of the EOS for the liquid are $\Gamma_{l}=7.14$ and $\frac{\Pi_{l}}{p_{b, 0}}=300$. Because even the compressible versions of the Rayleigh-Plesset equation (Keller-Miksis, Gilmore...) are only small modifications of the solution in the incompressible limit, it is important to correctly initialize the pressure field around the bubble to reproduce the conditions modelled in Rayleigh-Plesset models [24]. Given the pressure far from the bubble $p_{l, 0}$ the analytical pressure field for the Rayleigh collapse problem in the limit of an incompressible liquid is

$$
p(r, 0)=p_{l, 0}+\left(p_{l, 0}^{I}-p_{l, 0}\right) \frac{R_{0}}{r},
$$

where $p_{l, 0}^{I}=p_{l, 0}-\frac{2 \sigma}{R_{0}}$ is the initial liquid pressure at the interface given by Laplace's equation (Eq. 4). The cubic domain length is set to $L_{0} / R_{0}=100$ and the initial fluid velocity is set to zero everywhere.

Assuming spherical symmetry the Rayleigh collapse time is $t_{R}=0.915 R_{0} \sqrt{\frac{\rho_{l}}{\Delta p_{0}}}$, where $\Delta p_{0}=p_{l, 0}-p_{b, 0}$. In this work we use this time to define the characteristic velocity of the bubble collapse process, $U_{c}=0.915 R_{0} / t_{R}$ and to define the main non-dimensional quantities on which the problem depends: the pressure ratio $\frac{p_{l, 0}}{p_{b, 0}}$, the Weber number, We $=\frac{\Delta \mathrm{p}_{0} \mathrm{R}_{0}}{\sigma}$ the Reynolds number $\mathrm{Re}=\frac{\sqrt{\rho_{\mathrm{l}} \Delta \mathrm{p}_{0}} \mathrm{R}_{0}}{\mu_{1}}$. and the Mach number $\mathrm{Ma}=\frac{\mathrm{U}_{\mathrm{c}}}{\mathrm{c}_{1,0}}=\sqrt{\frac{\Delta \mathrm{p}_{0}}{\rho_{1} \mathrm{c}_{1,0}^{2}}}$.

We start investigating the collapse of a bubble in a viscous liquid at a moderate collapse intensity using the non-dimensional values indicated in 


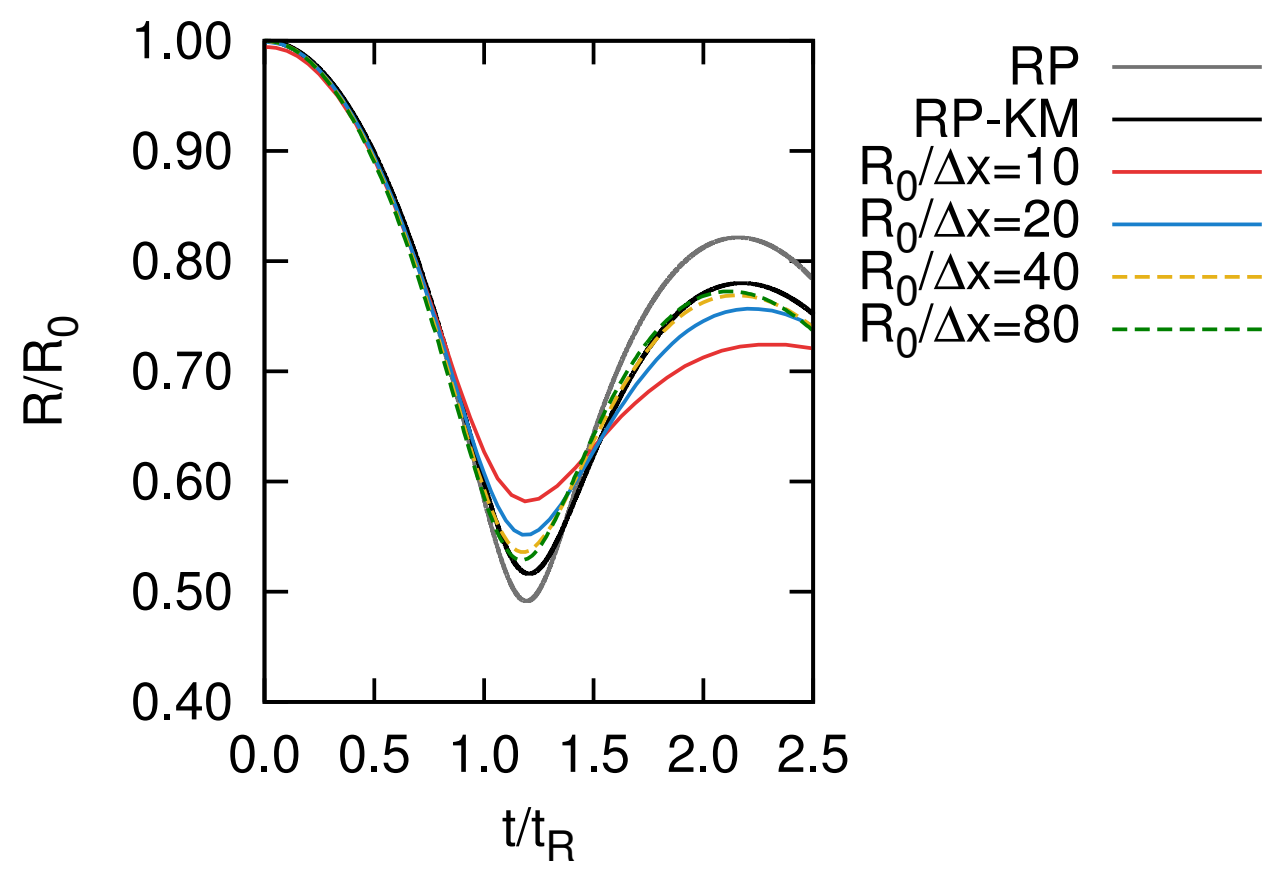

Figure 8: Temporal evolution of the bubble radius for the bubble collapse in a viscous fluid. SP: legend?

Table 1. Figure 8 shows the grid convergence of the temporal evolution of the effective radius $R=\left(\frac{V(t)}{4 / 3 \pi}\right)^{1 / 3}$ including as reference the solution obtained with a Rayleigh-Plesset model and the corrected solution provided by the Keller-Miksis equation [33]. Consistent with previous numerical results $[34,35]$, the numerical simulations tend to converge to a solution close to the prediction of the Keller-Miksis model. The collapse time matches well RP predictions showing very little sensitivity to the grid resolution. On the contrary, the amplitude of the rebound is significantly influenced by the grid resolution. Convergence is reached for a grid resolution of 40 points per bubble radius not only for the averaged bubble radius, but also for the amplitude of the non-spherical distortion computed from the standard deviation of the radial distance to the bubble center $\boldsymbol{x}_{c}$ of the centroid of all cells containing an interface (Figure 9). As predicted by the Rayleigh-Taylor 


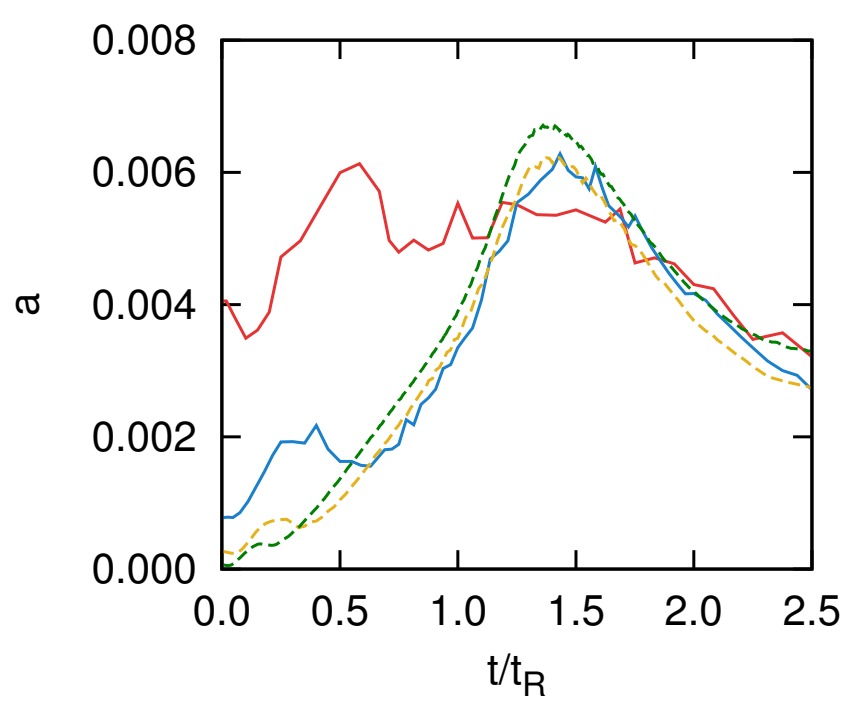

Figure 9: Temporal evolution of the radial disturbance $a$ for the bubble collapse in a viscous fluid.

$$
\begin{array}{ccccc}
p_{l, 0} / p_{b, 0} & r & \mathrm{Re} & \mathrm{We} & \mathrm{Ma} \\
\hline 20 & 10^{-3} & \infty & 1900 & 2.5 \times 10^{-2}
\end{array}
$$

Table 2: Non-dimensional paramters for the collapse of a bubble in an inviscid liquid used in Section 4.2.5.

instability models, the growth of non-spherical modes is promoted during the compression stage and also during the instants of possitive acceleration $\ddot{R}>0$. These modes are significantly damped during the expansion stage when deceleration occurs $\ddot{R}<0$, when the bubble recovers a nearly spherical shape at the end of the expansion after the rebound.

\subsubsection{Strongly non-linear collapse of a single bubble in an inviscid fluid}

In this section we test the solver for a strong collapse characterized by large values of the Weber and Reynolds numbers. The set of all nondimensional parameters is included in Table 2. Figure 10 presents the evolution of the effective bubble radius compared to the Keller-Miksis equation. 


\begin{tabular}{ccccc}
$p_{l, 0} / p_{b, 0}$ & $r$ & $\mathrm{Re}$ & $\mathrm{We}$ & $\mathrm{Ma}$ \\
\hline 40 & $10^{-3}$ & $\infty$ & 3800 & $2 \times 10^{-2}$
\end{tabular}

Table 3: Non-dimensional paramters for the collapse of a bubble close to a wall used in Section 4.2.6.

In this case viscous effects play a negligible role and liquid compressibility is the main mechanism responsible for damping and the resulting reduction of the amplitude of the collapse. The development of non-spherical instabilities at the interface during the collapse of the bubble is clearly seen in Figure 10 showing a local minimum at the moment of maximum radius during the rebound. The agreement with the Keller-Miksis solution is satisfactory during the overall process except on the amplitude of the rebound. We note that the Keller-Miksis solution is only an approximation of the full problem and previous numerical simulations solving the full compressible Navier-Stokes equations including compressibility effects and imposing spherical symmetry also show that the Keller-Miksis equation tends to overestimate the amplitude of the rebound [24].

\subsubsection{Non-linear collapse of a single bubble near a wall}

As a last example we show the collapse of a bubble near a wall. It is well known that the presence of any source of asymmetry leads to the appearance of a high speed jet during the collapse of the bubble [36, 37, 38]. The problem of the collapse near a wall has particular interest in various applications due to the consequences of jetting on the wall and it has motivated various experimental and numerical works (see for example [39, 40, 41]). In this work we consider the collapse of a single bubble placed at a distance $d / R_{0}=2$. In an attempt to reproduce the experimental results of Yang et al [41], we use the non-dimensional parameters included in Table 3 where the pressure ratio is obtained by assuming that the bubble pressure at the instant of maximum radius is approximately equal to the vapor pressure. The maximum resolution used in the region between the bubble and the wall is $R_{0} / \Delta x=40$.

Figure 11 depicts the bubble interface contour during different instants of the primary collapse with the pressure field in the middle plane and also the velocity field. The pressure increases during the instants of minimum radius 

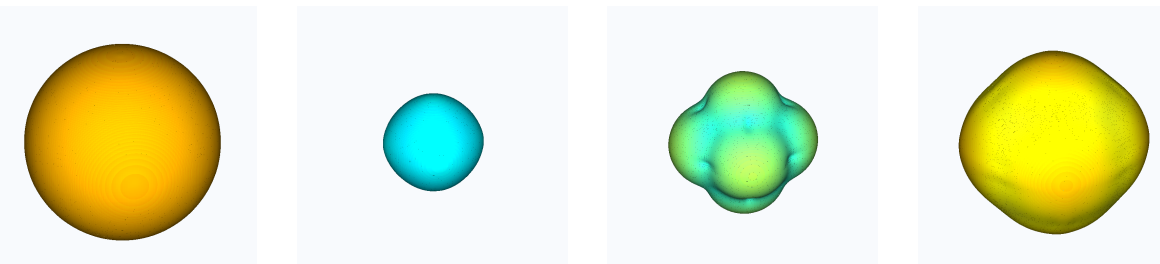

(a) $t / t_{R}=0.75$

(b) $t / t_{R}=1.00$

(c) $t / t_{R}=1.25$

(d) $t / t_{R}=1.50$

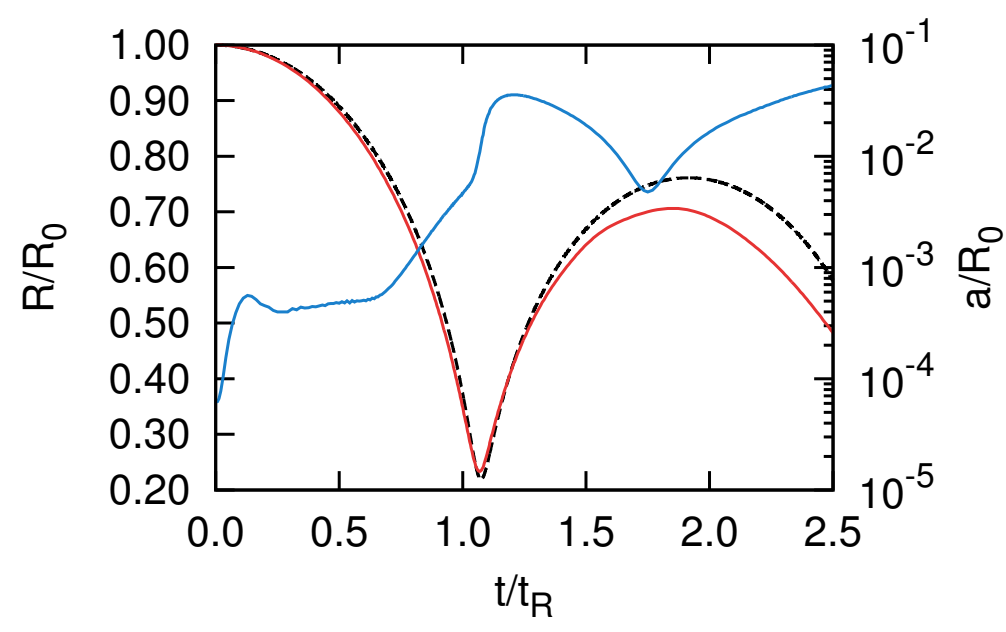

RP-KM --.-- R/R $\quad a / R_{0}$

Figure 10: Bubble collapse problem in the case of a strong collapse: $p_{l, 0} / p_{b, 0}=20$ and $\mathrm{We}=1900$. Top: $3 \mathrm{D}$ VOF surface colored as a function of the distance to the bubble center at $t / t_{R}=0.75,1.00,1.25,1.50$. The color scale range is $[0: 1]$. Bottom: Temporal evolution of the bubble radius (red) and standard deviation of the quantity $a / R_{0}=\sigma\left(\left|\boldsymbol{x}_{I}-\boldsymbol{x}_{c}\right| / R_{0}\right)$, which is a measure of the magnitude of the interface disturbance with respect to the averaged radius. 

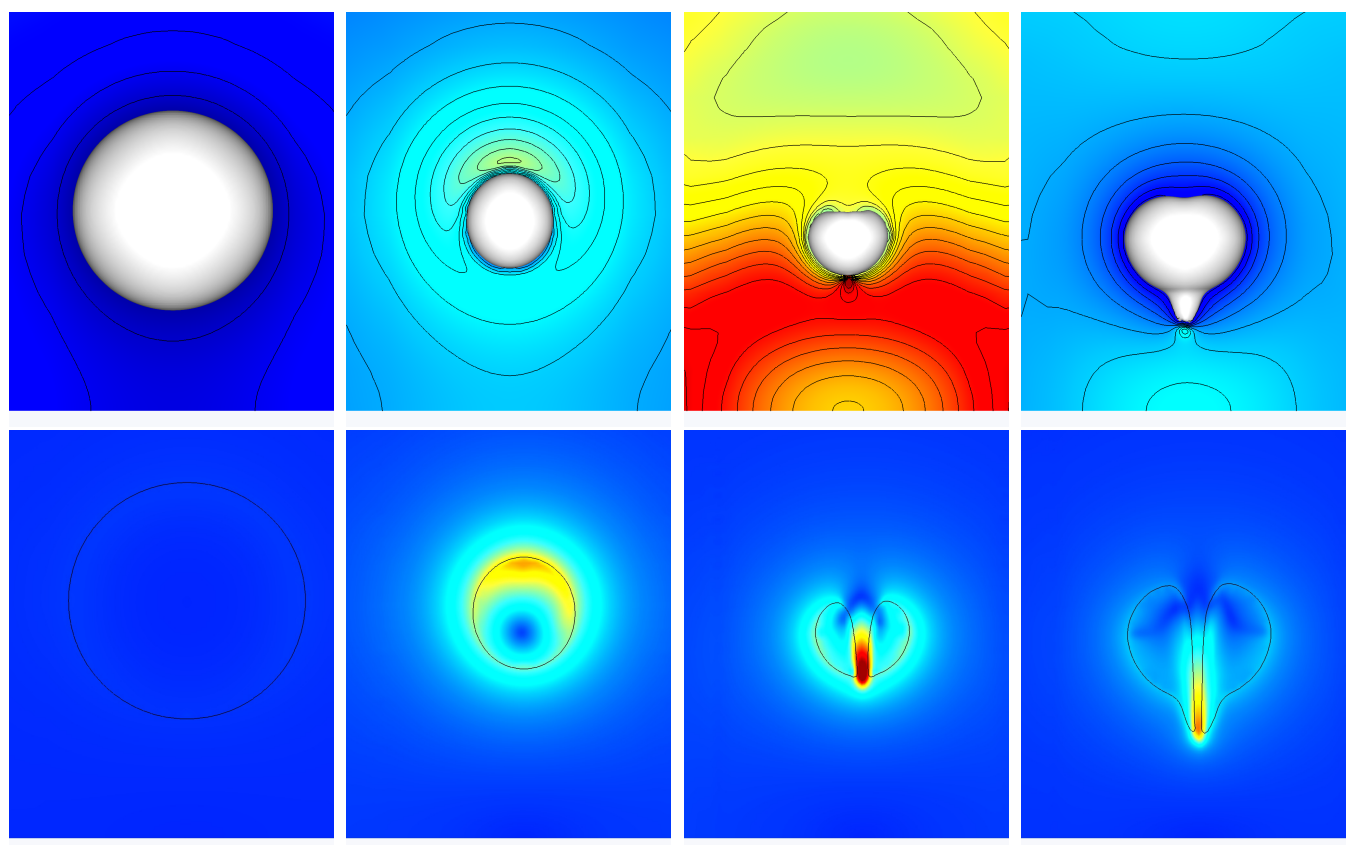

Figure 11: Bubble collapse near a wall (bottom boundary). From top to bottom: $t / t_{R}=$ 0, 0.96,1.20,1.40. Top: Bubble interface and pressure colormap (scale $p / p_{b, 0}=[1: 200]$ ) and isocontours on the middle plane. Bottom: Middle plane of the velocity color map (scale $|\boldsymbol{u}| t_{R} / R_{0}=[0: 3]$ ) and bubble interface. 

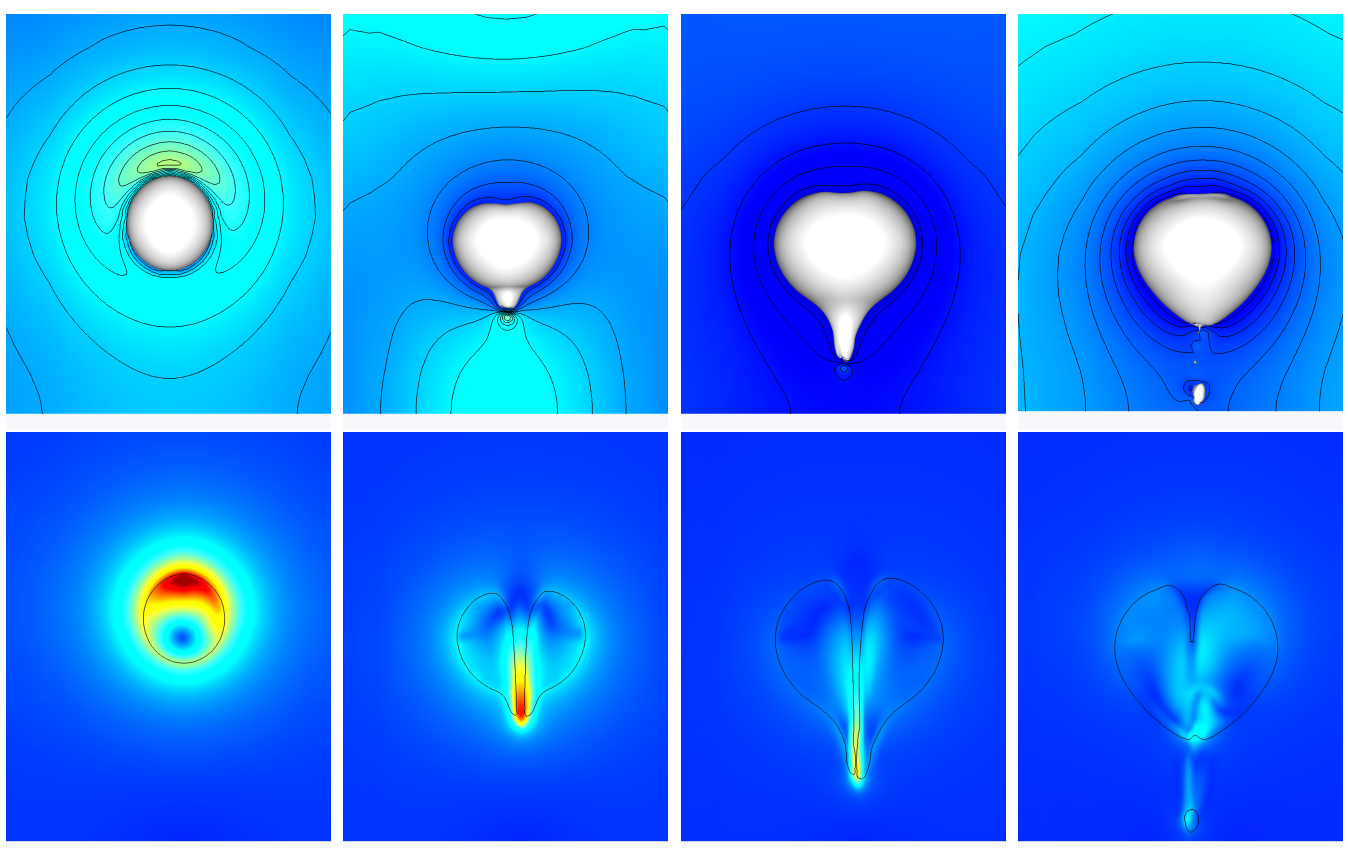

Figure 12: Evolution of the bubble shape and velocity fields for $t / t_{R}=0.96,1.30,1.65,2.20$. Same representation and ranges than those of Figure 11. 


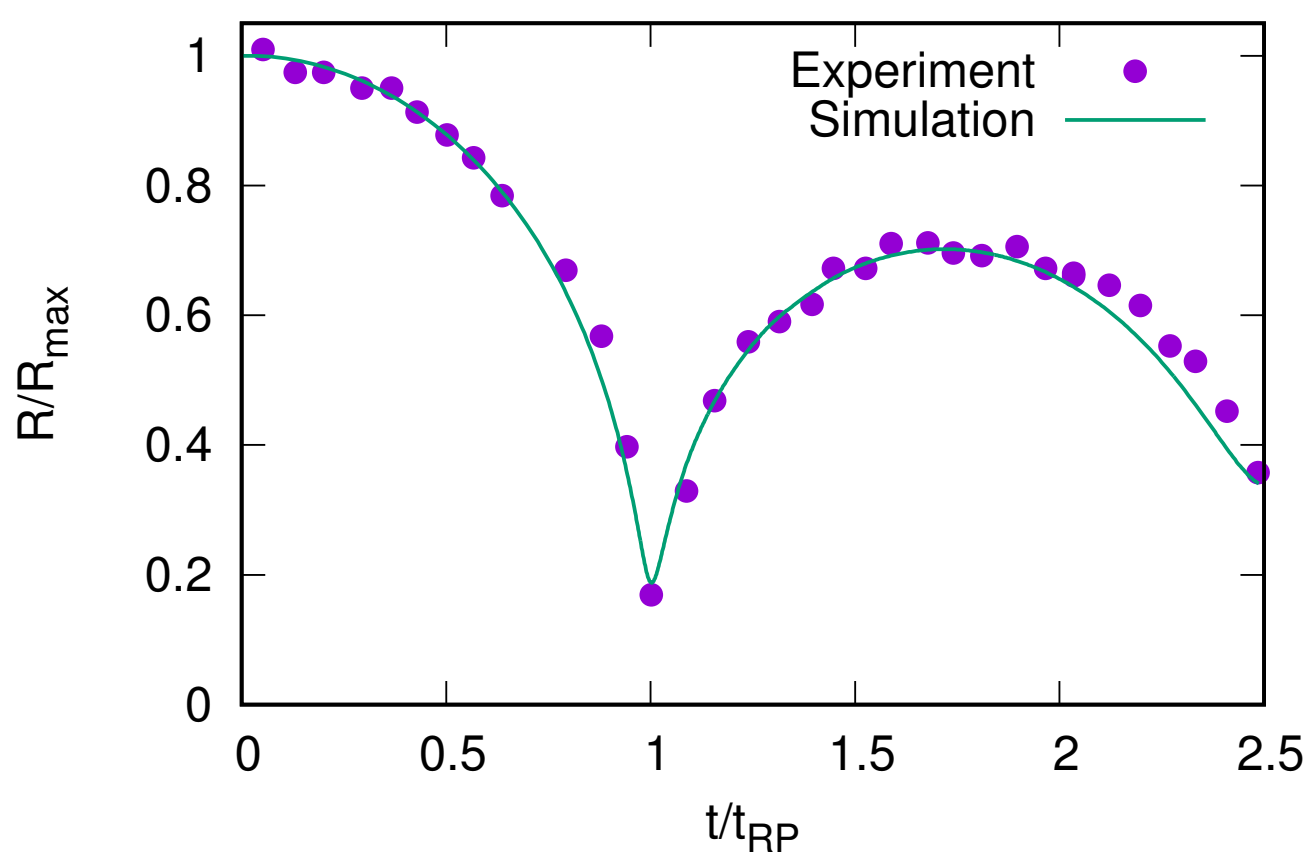

Figure 13: Evolution of the equivalent bubble radius close to a wall $\left(d / R_{0}=2\right)$ obtained from a full 3D simulation and comparison with the experimental results of Yang et al [41].

not only in the region surrounding the bubble but also in the wall. We can observe the generation of a high velocity liquid jet during the bubble collapse dragging some gas and impacting on the wall after the rebound (Figure 12). Figure 13 shows the evolution of the averaged bubble radius computed from the the total gas volume showing excellent agreement with the results reported by Yang et al [41] at an identical stand-off distance. The long term evolution of the bubble shape in a middle plane (Figure 14) shows similar features than those experimentally observed during the secondary collapse, namely the appearance of gas pockets that are left behind the jetting region due to the asymmetries generated by the presence of the wall, and the large deformation of the bubble shape for long times. 

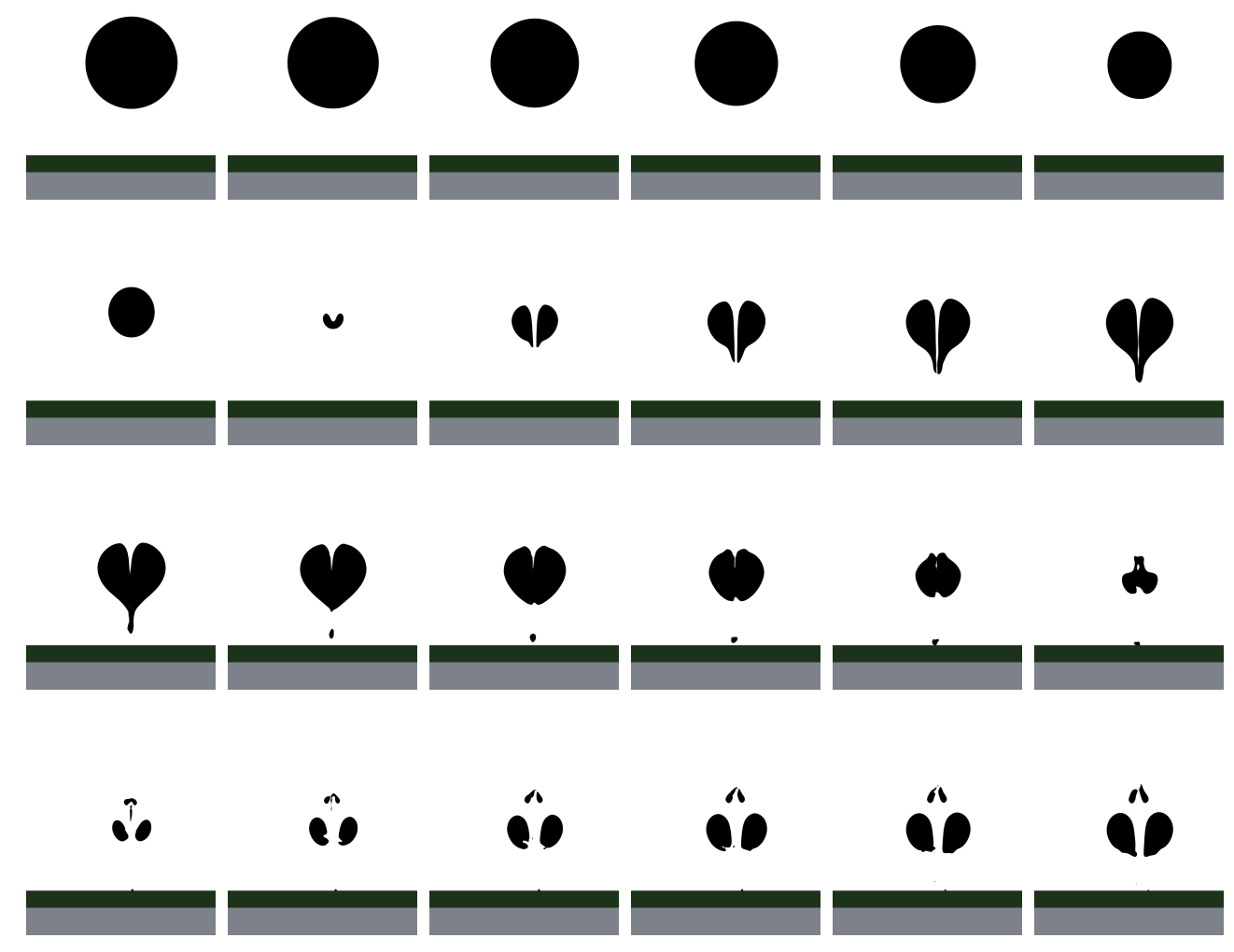

Figure 14: Evolution of the gas fraction in a middle plane for different instants during the collapse and later rebounds. Consistent with the experimental observations of Yang et al [41] gas pockets are left behind the jet during the secondary collapse and afterwards. 


\section{Conclusions}

In this manuscript we generalize an all-Mach semi-implicit formulation for the simulation of compressible multiphase flows including viscous and surface tension effects that also accounts for arbitrary values of the thermal dilatation coefficient. One of the particularities of the method is that the advection of conservative quantities is made consistent with the geometrical transport of the color function used in Volume-of-Fluid methods, thus avoiding any artificial transport of mass, momentum or energy between species. The method is fully conservative in the absence of viscous and surface tension forces which allows for the simulation of both linear wave propagation problems and problems involving shock waves.

The proposed formulation takes advantage of previous work developed in the context of incompressible solvers devoted to reducing the impact of spurious currents induced by surface tension forces and shows similar performances.

The method is tested in the context of the simulation of bubble oscillations in slightly compressible fluids. For linear oscillations, the method is shown to correctly predict the oscillation of the bubbles above and below the bubble resonance frequency. The nonlinear effects are discussed in the context of the Rayleigh collapse problem where we show grid convergence in a variety of situations involving surface tension and viscous effects. The results are consistent with the description of the physics of collapsing bubbles discussed in the literature. In particular we observe the appearance of non-spherical modes during the bubble collapse, typically attributed to the development of Rayleigh-Taylor instabilities, which are then damped during the bubble expansion .

The problem of the bubble collapse near a wall shows that, consistent with previous numerical and experimental observations, the method captures the generation of a high velocity jet that impacts on the wall leading to a significant bubble fragmentation. The method is shown to be robust even in the presence of small unresolved structures allowing to investigate the long term dynamic response of collapsing bubbles.

[1] R. H. Cole, R. Weller, Underwater explosions, Physics Today 1 (1948) 35. 
[2] Q. Wang, Non-spherical bubble dynamics of underwater explosions in a compressible fluid, Physics of Fluids 25 (7) (2013) 072104.

[3] T. Fourest, J.-M. Laurens, E. Deletombe, J. Dupas, M. Arrigoni, Analysis of bubbles dynamics created by hydrodynamic ram in confined geometries using the rayleigh-plesset equation, International Journal of Impact Engineering 73 (2014) 66-74.

[4] Q. Wang, K. Manmi, Three dimensional microbubble dynamics near a wall subject to high intensity ultrasound, Physics of Fluids 26 (3) (2014) 032104.

[5] V. Coralic, T. Colonius, Finite-volume weno scheme for viscous compressible multicomponent flows, Journal of Computational Physics 274 (2014) 95-121.

[6] K. Maeda, T. Colonius, W. Kreider, A. Maxwell, M. Bailey, Modeling and experimental analysis of acoustic cavitation bubble clouds for burst-wave lithotripsy, The Journal of the Acoustical Society of America 140 (4) (2016) 3307-3307.

[7] B. Hejazialhosseini, D. Rossinelli, M. Bergdorf, P. Koumoutsakos, High order finite volume methods on wavelet-adapted grids with local timestepping on multicore architectures for the simulation of shock-bubble interactions, Journal of Computational Physics 229 (22) (2010) 83648383 .

[8] K.-M. Shyue, F. Xiao, An eulerian interface sharpening algorithm for compressible two-phase flow: the algebraic thinc approach, Journal of Computational Physics 268 (2014) 326-354.

[9] P. Wesseling, Principles of computational fluid dynamics, Vol. 29, Springer Science \& Business Media, 2009.

[10] D. P. Garrick, M. Owkes, J. D. Regele, A finite-volume hllc-based scheme for compressible interfacial flows with surface tension, Journal of Computational Physics 339 (2017) 46-67.

[11] M. O. Abu-Al-Saud, S. Popinet, H. A. Tchelepi, A conservative and well-balanced surface tension model, Journal of Computational Physics. 
[12] S. Y. Yoon, T. Yabe, The unified simulation for incompressible and compressible flow by the predictor-corrector scheme based on the cip method, Computer Physics Communications 119 (2-3) (1999) 149-158.

[13] F. Xiao, Unified formulation for compressible and incompressible flows by using multi-integrated moments I: one-dimensional inviscid compressible flow, Journal of Computational Physics 195 (2) (2004) 629-654.

[14] N. Kwatra, J. Su, J. T. Grétarsson, R. Fedkiw, A method for avoiding the acoustic time step restriction in compressible flow, Journal of Computational Physics 228 (11) (2009) 4146-4161.

[15] F. Xiao, R. Akoh, S. Ii, Unified formulation for compressible and incompressible flows by using multi-integrated moments II: Multi-dimensional version for compressible and incompressible flows, Journal of Computational Physics 213 (1) (2006) 31-56.

[16] M. Jemison, M. Sussman, M. Arienti, Compressible, multiphase semiimplicit method with moment of fluid interface representation, Journal of Computational Physics 279 (2014) 182-217.

[17] J.-P. Caltagirone, S. Vincent, C. Caruyer, A multiphase compressible model for the simulation of multiphase flows, Computers \& fluids 50 (1) (2011) 24-34.

[18] S. Popinet, An accurate adaptive solver for surface-tension-driven interfacial flows, Journal of Computational Physics 288 (2009) 5838-5866.

[19] S. Popinet, A quadtree-adaptive multigrid solver for the "Serre-GreenNaghdi" equations, Journal of Computational Physics 302 (2015) 336358.

[20] D. Fuster, G. Agbaglah, C. Josserand, S. Popinet, S. Zaleski, Numerical simulation of droplets, bubbles and waves: state of the art, Fluid Dynamics Research 41 (2009) 065001.

[21] G. Tryggvason, R. Scardovelli, S. Zaleski, Direct numerical simulations of gas-liquid multiphase flows, Cambridge University Press, 2011.

[22] J. Brackbill, D. B. Kothe, C. Zemach, A continuum method for modeling surface tension, Journal of Computational Physics 100 (2) (1992) 335354. 
[23] L. Bergamasco, D. Fuster, Oscillation regimes of gas/vapor bubbles, International Journal of Heat and Mass Transfer 112 (2017) 72-80.

[24] D. Fuster, C. Dopazo, G. Hauke, Liquid compressibility effects during the collapse of a single cavitating bubble, Journal of the Acoustic Society of America 129 (1) (2011) 122-131.

[25] R. Saurel, R. Abgrall, A multiphase "Godunov" method for compressible multifluid and multiphase flows, Journal of Computational Physics 150 (2) (1999) 425-467.

[26] E. Johnsen, T. Colonius, Implementation of weno schemes in compressible multicomponent flow problems, Journal of Computational Physics 219 (2) (2006) 715-732.

[27] G. D. Weymouth, D. K.-P. Yue, Conservative volume-of-fluid method for free-surface simulations on cartesian-grids, Journal of Computational Physics 229 (8) (2010) 2853-2865.

[28] J. B. Bell, P. Colella, H. M. Glaz, A second-order projection method for the incompressible navier-stokes equations, Journal of Computational Physics 85 (2) (1989) 257-283.

[29] M. Rudman, A volume-tracking method for incompressible multifluid flows with large density variations, International Journal for Numerical Methods in Fluids 28 (2) (1998) 357-378.

[30] V. Le Chenadec, H. Pitsch, A monotonicity preserving conservative sharp interface flow solver for high density ratio two-phase flows, Journal of Computational Physics 249 (2013) 185-203.

[31] G. Vaudor, T. Ménard, W. Aniszewski, M. Doring, A. Berlemont, A consistent mass and momentum flux computation method for two phase flows. application to atomization process, Computers \& Fluids 152 (2017) 204-216.

[32] C. E. Brennen, Cavitation and bubble dynamics, Cambridge University Press, 2013.

[33] J. Keller, M. Miksis, Bubble oscillations of large amplitude, J. Acoust. Soc. Am. 68 (2) (1980) 628-633. 
[34] A. Tiwari, J. B. Freund, C. Pantano, A diffuse interface model with immiscibility preservation, Journal of computational physics 252 (2013) 290-309.

[35] M. Koch, C. Lechner, F. Reuter, K. Köhler, R. Mettin, W. Lauterborn, Numerical modeling of laser generated cavitation bubbles with the finite volume and volume of fluid method, using openfoam, Computers \& Fluids 126 (2016) 71-90.

[36] Q. Wang, W. Liu, A. Zhang, Y. Sui, Bubble dynamics in a compressible liquid in contact with a rigid boundary, Interface focus 5 (5) (2015) 20150048 .

[37] D. Obreschkow, M. Tinguely, N. Dorsaz, P. Kobel, A. De Bosset, M. Farhat, Universal scaling law for jets of collapsing bubbles, Physical review letters 107 (20) (2011) 204501.

[38] O. Supponen, D. Obreschkow, M. Tinguely, P. Kobel, N. Dorsaz, M. Farhat, Scaling laws for jets of single cavitation bubbles, Journal of Fluid Mechanics 802 (2016) 263-293.

[39] W. Lauterborn, H. Bolle, Experimental investigations of cavitationbubble collapse in the neighbourhood of a solid boundary, Journal of Fluid Mechanics 72 (2) (1975) 391-399.

[40] S. Popinet, S. Zaleski, Bubble collapse near a solid boundary: a numerical study of the influence of viscosity, Journal of Fluid Mechanics 464 (2002) 137-163.

[41] Y. X. Yang, Q. X. Wang, T. Keat, Dynamic features of a laser-induced cavitation bubble near a solid boundary, Ultrasonics sonochemistry 20 (4) (2013) 1098-1103. 